\title{
A First-Price Sealed-Bid Asymmetric Auction When Two Bidders Have Respective CRRA and General Utility Functions
}

\author{
Mingming Gong $\mathbb{i}$ and Shulin Liu $\mathbb{i}$ \\ School of International Trade and Economics, University of International Business and Economics, Beijing 100029, China \\ Correspondence should be addressed to Shulin Liu; slliu@uibe.edu.cn
}

Received 21 January 2021; Revised 24 July 2021; Accepted 18 August 2021; Published 6 September 2021

Academic Editor: Alfred Peris

Copyright (C) 2021 Mingming Gong and Shulin Liu. This is an open access article distributed under the Creative Commons Attribution License, which permits unrestricted use, distribution, and reproduction in any medium, provided the original work is properly cited.

We study a first-price auction with two bidders where one bidder is characterized by a constant relative risk aversion utility function (i.e., a concave power function) while the other has a general concave utility function. We establish the existence and uniqueness of the optimal strategic markups and analyze the effects of one bidder's risk aversion level on the optimal strategic markups of him and his opponent's, the allocative efficiency of the auction, and the seller's expected revenue, respectively.

\section{Introduction}

Though the rules of a first-price auction are simple, the same cannot necessarily be said for the case that bidders are asymmetric, especially when the bidders are risk averse. Previous studies on the asymmetric auctions with risk neutral bidders within the independent private value (IPV) model have been extensively studied by Lebrun [1], Maskin and Riley [2, 3], Kirkegaard [4], and Kaplan and Zamir [5]. Also, the symmetric auctions with risk-averse preferences have been much studied in the literature (e.g., Riley and Samuelson [6], Matthews [7], and $\mathrm{Hu}$ et al. [8]). In this paper, we build on the model outlined by Maréchal and Morand [9] (henceforth MM) and study first-price sealedbid auctions with asymmetric bidders in terms of risk aversion.

The MM's model is initiated by Von Ungern-Sternberg [10] (henceforth VUS) in his theoretical analysis on Swiss construction industry of simultaneous sealed-bid multiobject auctions, where each bidder are ex ante symmetric relative to the informational knowledge and he can predict his private valuation with certainty but has no grounds for believing it to be higher or lower on average than his opponent's. Formally, VUS models this by assuming that the different bidders' valuations are independent drawings from a uniform distribution with an unknown mean; thereby, the bidders can use this information to infer the mean and indirectly infer his opponent's valuation. Therefore, the ex ante and ex post distribution are not the same, which is the main difference between the standard IPV model and the VUS model.

MM follows the VUS model and extends it to the case of two risk-averse bidders who had the same parametric family of (CRRA) functions, but their measures of risk aversion differ. Then, they derive the explicit expressions of asymmetric bidding equilibrium. To the best of our knowledge, it seems that only Maréchal and Morand consider asymmetric first-price auctions where two bidders assume different CRRA utility functions. By allowing risk aversion levels of both bidders to vary simultaneously, they show that as one bidder becomes less risk averse and the other one becomes more risk averse, (i) the less risk-averse bidder reduces his markup when asymmetry in terms of risk aversion between both bidders is sufficiency large; (ii) the allocative efficiency decreases when asymmetry increases; and (iii) the seller's expected revenue increases as bidders become more asymmetric in terms of risk aversion.

We generalize the work by MM to a more general case where one bidder is characterized by a CRRA utility function while the other has a general concave utility function. Our work makes a contribution to the asymmetric auction literature and is different from MM in several respects: (i) we 
establish the existence and uniqueness of the optimal strategic markups of two bidders (see Propositions 1 and 2); (ii) we study effects of one bidder's risk aversion on bidding behavior. We find that when one bidder becomes more risk averse and the other bidder's risk averse remain unchanged, both bidders reduce their optimal strategic markups, and an illustrative example shows that the rate at which his markup decreases is higher than the rate at which his opponent's markup decreases (see Propositions 3 and 4); (iii) the allocative efficiency becomes complicated as one bidder becomes more (less) risk averse (see our Proposition 6); (iv) we study effects of one bidder's risk aversion on expected revenue. We find that the seller's expected revenue increases when one bidder becomes more risk averse, given that the other bidder's risk averse remain unchanged (see Proposition 7). Section 4 concludes the paper.

\section{Model}

In this paper, we study a two-bidder first-price sealed-bid auction selling an indivisible single item whereas adopting the framework of the model as in MM. Differentiating from their framework, we further generalize the MM model by accommodating the case where one bidder has a general concave utility function and the other still has a CRRA utility function.

2.1. The MM's Model. In MM, the key assumptions conclude the following: (a) both bidders are ex ante symmetric relative to the informational knowledge; (b) each bidder has a private valuation; (c) the valuations are independently drawn from a known uniform distribution $F$ and distributes around the unknown mean $\mu$ over $[\mu-a, \mu+a]$ with frequency $1 / 2 a$, where $a$ is common knowledge; (d) the optimal strategy is to bid a simple markup $b$ below his valuation and the markup $b$ is independent of his valuation.

Such as bidder $\alpha$, when he observes his own valuation $v_{\alpha}$, since assumption (c), he can draw inferences about $\mu \in\left[v_{\alpha}-a, v_{\alpha}+a\right]$ according to the cumulative $F_{\mu}$ with corresponding density $f_{\mu}$. Due to assumption (a), he can indirectly draw inferences about his opponent's valuation $v_{\beta} \in\left[v_{\alpha}-2 a, v_{\alpha}+2 a\right]$ according to the cumulative $F_{\beta}$ with corresponding density $f_{\beta}$.

Suppose bidder $i \in\{\alpha, \beta\}$ has a private valuation of $v_{i}$ and places a bid $B_{i}$. Due to assumption (d), the bid has the form $B_{i}\left(v_{i}\right)=v_{i}-b_{i}$. When his competitor chooses a markup equal to $b_{-i}$ (-i means $i$ 's opponent), bidder $i$ 's probability of winning $\operatorname{Prob}_{i}$ (win) is given by

$$
\int_{\mu} \operatorname{prob}\left(B_{i}>B_{-i}\right) f_{\mu}(\mu) \mathrm{d} \mu .
$$

Consider bidder $\alpha$, the probability of winning $P$ is

$$
P=\int_{\mu} F_{\beta}\left(v_{\alpha}-b_{\alpha}+b_{\beta}\right) f_{\mu}(\mu) \mathrm{d} \mu, \quad \mu \in\left[v_{\alpha}-a, v_{\alpha}+a\right] .
$$

Then, we have

$$
P=\int_{\mu} \frac{1}{2 a}\left(\frac{a-b_{\alpha}+b_{\beta}+v_{\alpha}-\mu}{2 a}\right) \mathrm{d} \mu .
$$

$Q$ is

Similarly, consider bidder $\beta$, the probability of winning

$$
Q=\int_{\mu} F_{\alpha}\left(v_{\beta}-b_{\beta}+b_{\alpha}\right) f_{\mu}(\mu) \mathrm{d} \mu, \quad \mu \in\left[v_{\beta}-a, v_{\beta}+a\right] .
$$

Then, we have

$$
Q=\int_{\mu} \frac{1}{2 a}\left(\frac{a+b_{\alpha}-b_{\beta}+v_{\beta}-\mu}{2 a}\right) \mathrm{d} \mu .
$$

2.2. The General Utility Function of Bidders. Bidders are risk averse and one bidder has a CRRA utility function $u_{i}(x)=x^{\rho_{i}}$ (with $0<\rho_{i} \leq 1$ ), while the other bidder $-i$ has a general utility function $u_{-i}(x)$ satisfying $u_{-i}(0)=0$, $u_{-i}^{\prime}(x)>0$, and $u_{-i}^{\prime \prime}(x) \leq 0, x$ is bidder's income. Specifically, we consider two models as shown in the following:

Model I: suppose that bidder $\alpha$ has a utility function $u_{\alpha}(x)=x^{\rho_{\alpha}}$ (with $\left.0<\rho_{\alpha} \leq 1\right)$ and bidder $\beta$ has a general utility function $u_{\alpha}(x)$ satisfying $u_{\alpha}(0)=0, u_{\alpha}^{\prime}(x)>0$, and $u_{\alpha}^{\prime \prime}(x) \leq 0$. Then, the respective expected utility maximization problems for bidders $\alpha$ and $\beta$ are

$$
\begin{aligned}
& \max _{b_{\alpha}} E u_{\alpha}=\left(b_{\alpha}\right)^{\rho_{\alpha}} P\left(b_{\alpha}, b_{\beta}\right), \\
& \max _{b_{\beta}} E u_{\beta}=u_{\beta}\left(b_{\beta}\right) Q\left(b_{\alpha}, b_{\beta}\right),
\end{aligned}
$$

where $P\left(b_{\alpha}, b_{\beta}\right)$ and $Q\left(b_{\alpha}, b_{\beta}\right)$ are the respective probabilities of winning of bidder $\alpha$ and $\beta$ when they choose strategic markups $b_{\alpha}$ and $b_{\beta}$,

$$
\begin{aligned}
P\left(b_{\alpha}, b_{\beta}\right) & =\frac{\left(2 a-b_{\alpha}+b_{\beta}\right)^{2}}{8 a^{2}}, \\
Q\left(b_{\alpha}, b_{\beta}\right) & =\frac{4 a^{2}+4 a\left(b_{\alpha}-b_{\beta}\right)-\left(b_{\alpha}-b_{\beta}\right)^{2}}{8 a^{2}} .
\end{aligned}
$$

Model II: suppose that bidder $\alpha$ has a general utility function $u_{\alpha}(x)$ and bidder $\beta$ has a utility function $u_{\beta}(x)=x^{\rho_{\beta}}$ (with $0<\rho_{\beta} \leq 1$ ) (see page 109 of MM for the detailed proof). Then, the respective expected utility maximization problems for bidders $\alpha$ and $\beta$ are

$$
\begin{aligned}
& \max _{b_{\alpha}} E u_{\alpha}=u_{\alpha}\left(b_{\alpha}\right) P\left(b_{\alpha}, b_{\beta}\right), \\
& \max _{b_{\beta}} E u_{\beta}=\left(b_{\beta}\right)^{\rho_{\beta}} Q\left(b_{\alpha}, b_{\beta}\right),
\end{aligned}
$$

where $b_{\alpha}, b_{\beta}, P\left(b_{\alpha}, b_{\beta}\right)$, and $Q\left(b_{\alpha}, b_{\beta}\right)$ have the same meanings as in Model I.

Define $\gamma_{i}=u_{i} / u_{i}^{\prime}$. Let $R_{i}=-u_{i}^{\prime \prime} / u_{i}^{\prime}$ denote the ArrowPratt measure of absolute risk aversion. Since 
$\dot{\gamma}_{i}=\left[\left(\dot{u}_{i}^{\prime}\right)^{2}-u_{i} u_{i}^{\prime \prime}\right] /\left(\dot{u}_{i}^{\prime}\right)^{2}=1-\left(\gamma_{i} u_{i}^{\prime \prime}\right) / \dot{u}_{i}^{\prime}, \gamma_{i}$ is related to $R_{i}$ by $\gamma_{i}^{\prime}=1+R_{i} \gamma_{i}$. Since $\gamma_{i}(x) \geq 0$ and $R_{i}(x) \geq 0$ for $x \geq 0$, $\hat{\gamma}_{i}^{\prime}(x)>0$ for $x \geq 0$. Let $\widehat{u}_{i}$ be another utility function of bidder $i$ satisfying the same assumptions as $u_{i}$, with an absolute risk aversion measure $\widehat{R}_{i}(x)$ such that $\widehat{R}_{i}(x)>R_{i}(x)$ on $(0, \infty]$. Then, $\widehat{R}_{i}(x)>R_{i}(x)$ on $(0, \infty)$ implies $\widehat{\gamma}_{i}(x)>\gamma_{i}(x)$ on $(0$, $\infty)$ (see page 1191 of $\mathrm{Hu}$ et al. [8]).

Throughout the paper, we keep the assumption of $b_{\alpha} \geq b_{\beta}$. (Similarly, as in MM, the two probabilities given in (8) and (9) will be changed when we assume that $b_{\alpha} \leq b_{\beta}$. With this assumption, we can get results that are entirely parallel to the existing propositions in Section 3.) That is used to derive the two probabilities of winning of (8) and (9) as in MM. The following examples verify this assumption.

Example 1. Suppose that bidder $\alpha$ has a CRRA utility function $u_{\alpha}(x)=x^{\rho_{\alpha}}$ with $0<\rho_{\alpha} \leq 1$, and bidder $\beta$ has a constant absolute risk aversion (CARA) utility function $u_{\beta}$ $(x)=1-\exp \left(-\theta_{\beta} x\right)$ with $\theta_{\beta}>0$. Substituting $u_{\beta}(x)$ into (15) yields

$$
\frac{-1+e^{\theta_{\beta} b_{\beta}}}{\theta_{\beta}}=\frac{2 a^{2}\left(\rho_{\alpha}+2\right)}{b_{\beta}+2 a}-\frac{b_{\beta}+2 a}{\rho_{\alpha}+2} .
$$

This and (14) constitute a system. Let $a=1$. Solving the respective six systems associated with six pairs of $\left(\rho_{\alpha}, \theta_{\beta}\right)$, we obtain six pairs of optimal markups as shown in Table 1.

Table 1 shows the optimal markups satisfy $b_{\alpha} \geq b_{\beta}$.

Example 2. Suppose that bidder $\alpha$ has a CARA utility function $u_{\alpha}(x)=1-\exp \left(-\theta_{\alpha} x\right)$ with $\theta_{\alpha}>0$, and bidder $\beta$ has a CRRA utility function $u_{\beta}(x)=x^{\rho_{\beta}}$ with $0<\rho_{\beta} \leq 1$. Substituting $u_{\alpha}(x)$ into (18) yields

$$
\frac{-1+e^{\theta_{\alpha} b_{\alpha}}}{\theta_{\alpha}}=\frac{2 a-b_{\alpha}+\sqrt{\left(2 a-b_{\alpha}\right)^{2}+8 a^{2}\left(\rho_{\beta}^{2}+2 \rho_{\beta}\right)}}{2\left(2+\rho_{\beta}\right)} .
$$

This and (17) constitute a system. Let $a=1$. Solving the respective six systems associated with six pairs of $\left(\theta_{\alpha}, \rho_{\beta}\right)$ as shown in the following Table 2, we obtain six pairs of optimal markups.

Table 2 shows that the optimal markups satisfy $b_{\alpha} \geq b_{\beta}$.

\section{Optimal Strategic Markups and Their Properties}

\subsection{Existence of Optimal Strategic Markups}

\section{Proposition 1. Consider Model I. Then,}

(i) The optimal strategic markups for both bidders $\left(b_{\alpha}\right.$, $b_{\beta}$ ) are characterized by (when $u_{\beta}(x)=x^{\rho_{\beta}}$, we get the same optimal strategic markups as in $M M$ )

$$
\begin{aligned}
b_{\alpha} & =\frac{\rho_{\alpha}\left(2 a+b_{\beta}\right)}{2+\rho_{\alpha}}, \\
\gamma_{\beta}\left(b_{\beta}\right) & =G\left(b_{\beta}\right),
\end{aligned}
$$

where
TABLE 1: Verification of the assumption $b_{\alpha} \geq b_{\beta}$.

\begin{tabular}{ccccccc}
\hline$\rho_{\alpha}$ & 0.8 & 0.8 & 0.9 & 0.9 & 0.9 & 0.9 \\
\hline$\theta_{\beta}$ & 0.6 & 0.7 & 0.3 & 0.7 & 0.8 & 0.9 \\
$b_{\alpha}$ & 0.7971 & 0.7926 & 0.8955 & 0.8726 & 0.8674 & 0.8624 \\
$b_{\beta}$ & 0.7900 & 0.7743 & 0.8858 & 0.8118 & 0.7951 & 0.7790 \\
\hline
\end{tabular}

TABLE 2: Verification of the assumption $b_{\alpha} \geq b_{\beta}$.

\begin{tabular}{ccccccc}
\hline$\theta_{\alpha}$ & 0.2 & 0.3 & 0.35 & 0.4 & 0.41 & 0.8 \\
\hline$\rho_{\beta}$ & 0.7 & 0.8 & 0.8 & 0.8 & 0.8 & 0.2 \\
$b_{\alpha}$ & 0.8762 & 0.8635 & 0.8492 & 0.8354 & 0.8328 & 0.6400 \\
$b_{\beta}$ & 0.7915 & 0.8347 & 0.8269 & 0.8194 & 0.8179 & 0.3114 \\
\hline
\end{tabular}

$$
G(x)=\frac{2 a^{2}\left(\rho_{\alpha}+2\right)}{x+2 a}-\frac{x+2 a}{\rho_{\alpha}+2}, \quad \text { on }[0, \infty) .
$$

(ii) There exists a unique optimal strategic markup ( $b_{\alpha}$ $\left.b_{\beta}\right)$ with $b_{\alpha}, b_{\beta} \in\left(0, a \rho_{\alpha}\right]$.

(iii) $b_{\alpha}$ and $b_{\beta}$ increase with the uncertainty parameter $a$.

Proof. See Appendix.

Part (iii) of Proposition 1 is in line with intuition and conventional results in auction theory (see among others Klemperer [11] and Krishna [12]).

Proposition 2. Consider Model II. Then,

(i) The optimal strategic markups for both bidders $\left(b_{\alpha}\right.$, $\left.b_{\beta}\right)$ are characterized by $\left(\right.$ when $u_{\alpha}(x)=x^{\rho_{\alpha}}$, we have the same optimal strategic markups as in $M M$ )

$$
\begin{aligned}
b_{\beta} & =g\left(b_{\alpha}\right), \\
\gamma_{\alpha}\left(b_{\alpha}\right) & =L\left(b_{\alpha}\right),
\end{aligned}
$$

where $g(x)$ and $L(x)$ are defined on $[0, \infty)$,

$$
g(x)=\frac{\left(1+\rho_{\beta}\right)(x-2 a)+\sqrt{(2 a-x)^{2}+8 a^{2}\left(\rho_{\beta}^{2}+2 \rho_{\beta}\right)}}{2+\rho_{\beta}},
$$

$$
L(x)=\frac{2 a-x+\sqrt{(2 a-x)^{2}+8 a^{2}\left(\rho_{\beta}^{2}+2 \rho_{\beta}\right)}}{2\left(2+\rho_{\beta}\right)} .
$$

(ii) There exists a unique optimal strategic markup $\left(b_{\alpha}\right.$ $\left.b_{\beta}\right)$ with $b_{\alpha}, b_{\beta} \in\left[a \rho_{\beta}, a\right)$.

(iii) $b_{\alpha}$ and $b_{\beta}$ are increasing with respect to the uncertainty parameter $a$.

Proof. See Appendix.

3.2. Impact of the Degrees of Risk Aversion on Bidders' Optimal Markups. Suppose that bidder $\alpha$ 's risk aversion level remains unchanged. Then, Proposition 3 (i) shows that the 
optimal strategic markups for both bidders $\alpha$ and $\beta$ decrease in risk aversion of bidder $\beta$. Part (ii) shows that a change in bidder $\beta$ 's optimal strategic markup is greater than a change in bidder $\alpha$ 's optimal strategic markup as bidder $\beta$ becomes more risk averse.

Proposition 3. Consider Model I. Let $b_{\alpha}$ and $b_{\beta}$ be the optimal strategic markups for bidder $\alpha$ and $\beta$ associated with utility functions $u_{\alpha}(x)=x^{\rho_{\alpha}}$ and $u_{\beta}(x)$, satisfying that $b_{\alpha} \geq b_{\beta}$. Let $\widehat{b}_{\alpha}$ and $\widehat{b}_{\beta}$ be the optimal strategic markups for both bidders associated with utility functions $u_{\alpha}(x)=x^{\rho_{\alpha}}$ and $\widehat{u}_{\beta}$, satisfying that $\widehat{b}_{\alpha} \geq \widehat{b}_{\beta}$. Then, we have the following:

$$
\begin{aligned}
& \text { (i) } \widehat{b}_{\alpha}<b_{\alpha}, \widehat{b}_{\beta}<b_{\beta} \\
& \text { (ii) } b_{\alpha}-\widehat{b}_{\alpha}<b_{\beta}-\widehat{b}_{\beta} \\
& \text { (i.e., } \left.\left(v_{\alpha}-b_{\alpha}\right)-\left(v_{\alpha}-b_{\alpha}\right)<\left(v_{\beta}-\widehat{b}_{\beta}\right)-\left(v_{\beta}-b_{\beta}\right)\right)
\end{aligned}
$$

Proof. See Appendix.

The following Examples 3 and 4 illustrate the influence of the degrees of bidder $\beta$ 's risk aversion on the markups of both bidders.

Example 3. Consider Example 1 again. Suppose that bidder $\beta$ becomes more risk averse. Let $\theta_{\beta}$ become $\theta_{\beta}+\varepsilon$, where $\varepsilon$ is a positive real number. Then, the optimal strategic markups $b_{\alpha}$ and $b_{\beta}$ satisfy

$$
\begin{gathered}
\frac{-1+e^{\left(\theta_{\beta}+\varepsilon\right) \widehat{b}_{\beta}}}{\left(\theta_{\beta}+\varepsilon\right)}=\frac{2 a^{2}\left(\rho_{\alpha}+2\right)}{\widehat{b}_{\beta}+2 a}-\frac{\widehat{b}_{\beta}+2 a}{\rho_{\alpha}+2}, \\
\widehat{b}_{\alpha}=\frac{\rho_{\alpha}\left(2 a+\widehat{b}_{\beta}\right)}{2+\rho_{\alpha}}, \\
\widehat{b}_{\alpha}, \widehat{b}_{\beta}\left(0, \alpha \rho_{\alpha}\right] .
\end{gathered}
$$

Figure 1 depicts the optimal strategic markups $b_{\alpha}$ and $b_{\beta}$ for $\varepsilon \in(0,0.1)$ (the choice of $\varepsilon$ 's range must guarantee the optimal strategic markups exist in the interval $\left(0, a \rho_{\alpha}\right)$, where $a=1, \theta_{\beta}=0.6$, and $\rho_{\alpha}=0.8$. It shows that both optimal strategic markups are declining with $\varepsilon$, implying that as bidder $\beta$ becomes more risk averse, both $b_{\alpha}$ and $b_{\beta}$ decrease (i.e., part (i) of Proposition 3 holds), $b_{\beta}$ is more rapidly decreased than $b_{\alpha}$, and the magnitude of the asymmetry effects on the optimal bid markup becomes smaller for bidder $\alpha$ but becomes bigger for bidder $\beta$. It also shows that the magnitude of the asymmetry effects is less for bidder $\alpha$ than that for bidder $\beta$ (i.e., part (i) of Proposition 3 holds).

Example 4. Consider that both bidders exhibit constant relative risk aversion (CRRA) utility functions, $u_{i}(x)=x^{\rho_{i}}$ (with $0<\rho_{i} \leq 1, i \in\{\alpha, \beta\}$ ), where $1-\rho_{i}$ is the Arrow-Pratt measure of CRRA. Then, we can derive explicitly the optimal strategic markups for both bidders

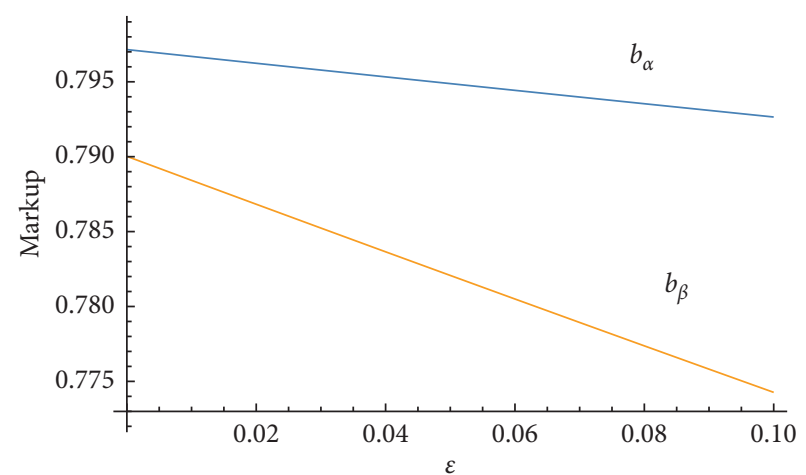

FIgURE 1: Plot of the optimal strategic markups $b_{\alpha}$ and $b_{\beta}$ increase with the degrees of bidder $\beta$ 's risk aversion.

$$
\begin{aligned}
& b_{\alpha}=\frac{a \rho_{\alpha}(1+A)}{2+\rho_{\alpha}+\rho_{\beta}}, \\
& b_{\beta}=\frac{a\left(\left(2+\rho_{\alpha}\right) A-2-\rho_{\alpha}-2 \rho_{\beta}\right)}{2+\rho_{\alpha}+\rho_{\beta}},
\end{aligned}
$$

where $A=\sqrt{1+2 \rho_{\beta}\left(2+\rho_{\alpha}+\rho_{\beta}\right)}$.

Suppose that bidder $\beta$ becomes more risk averse. Let $\rho_{\beta}$ become $\rho_{\beta}-\delta$, where $\delta$ is a positive real number. Then, we have

$$
\begin{aligned}
& \widehat{b}_{\alpha}=\frac{a \rho_{\alpha}(1+\widehat{A})}{2+\rho_{\alpha}+\rho_{\beta}-\delta}, \\
& \widehat{b}_{\beta}=\frac{a\left(\left(2+\rho_{\alpha}\right) \widehat{A}-2-\rho_{\alpha}-2\left(\rho_{\beta}-\delta\right)\right)}{2+\rho_{\alpha}+\rho_{\beta}-\delta},
\end{aligned}
$$

where $\widehat{A}=\sqrt{1+2\left(\rho_{\beta}-\delta\right)\left(2+\rho_{\alpha}+\rho_{\beta}-\delta\right)}$.

Figure 2 depicts the optimal strategic markups $b_{\alpha}$ and $b_{\beta}$ for $\delta \in(0,0.55)$, where $a=1, \rho_{\alpha}=0.9$, and $\rho_{\beta}=0.6$. It shows that both optimal strategic markups are declining with $\delta$, implying that as bidder $\beta$ becomes more risk averse, both $b_{\alpha}$ and $b_{\beta}$ decrease (i.e., part (i) of Proposition 3 holds), $b_{\beta}$ is more rapidly decreased than $b_{\alpha}$, and the magnitude of the asymmetry effects on the optimal bid markup becomes smaller for bidder $\alpha$ but becomes bigger for bidder $\beta$. It also shows that the magnitude of the asymmetry effects is less for bidder $\alpha$ than that for bidder $\beta$ (i.e., part (ii) of Proposition 3 holds).

Suppose that bidder $\beta$ 's risk aversion level remains unchanged. When bidder $\alpha$ becomes more risk averse, Proposition 4 has a similar meaning as Proposition 3.

Proposition 4. Consider Model II. Let $b_{\alpha}$ and $b_{\beta}$ be optimal strategic markups for bidder $\alpha$ and $\beta$ associated with $u_{\alpha}(x)$ and $u_{\beta}(x)=x^{\rho_{\beta}}$, satisfying that $b_{\alpha} \geq b_{\beta}$. Let $\bar{b}_{\alpha}$ and $\bar{b}_{\beta}$ be the optimal strategic markups for both bidders associated with utility functions $u_{\beta}(x)=x^{\rho_{\beta}}$ and $\widehat{u}_{\alpha}$, satisfying that $\bar{b}_{\alpha} \geq \bar{b}_{\beta}$. Then, we have the following:

$$
\text { (i) } \bar{b}_{\alpha}<b_{\alpha}, \bar{b}_{\beta}<b_{\beta}
$$




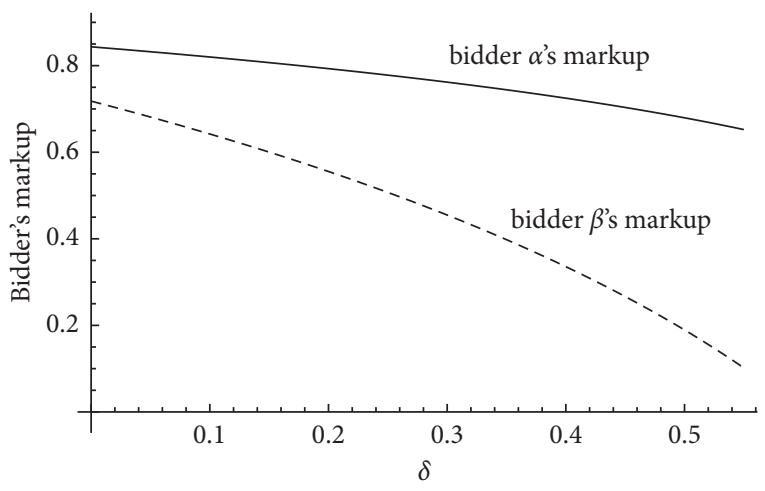

Figure 2: Plot of the optimal strategic markups $b_{\alpha}$ and $b_{\beta}$ increase with the degree of bidder $\beta$ 's risk aversion.

(ii) $b_{\beta}-\bar{b}_{\beta}<b_{\alpha}-\bar{b}_{\alpha}$ (i.e., $\left(v_{\beta}-\bar{b}_{\beta}\right)-\left(v_{\beta}-b_{\beta}\right)<\left(v_{\alpha}-\right.$ $\left.\left.\vec{b}_{\alpha}\right)-\left(v_{\alpha}-b_{\alpha}\right)\right)$

Proof. See Appendix.

The following Examples 5 and 6 illustrate the influence of the degrees of bidder $\alpha$ 's risk aversion on the markups of both bidders.

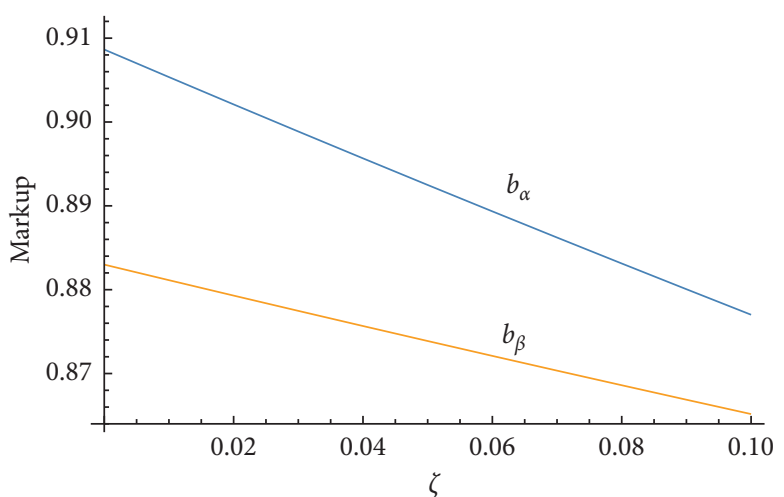

FIgURe 3: Plot of the optimal strategic markups $b_{\alpha}$ and $b_{\beta}$ increase with the degree of bidder $\alpha$ 's risk aversion.

Example 5. Consider Example 2 again. Suppose that bidder $\alpha$ becomes more risk averse. Let $\theta_{\alpha}$ becomes $\theta_{\alpha}+\zeta$, where $\zeta$ is a positive real number, and the larger $\zeta$ is, the bidder $\alpha$ becomes more risk averse. Then, the optimal strategic markups satisfy

$$
\left\{\begin{array}{l}
\frac{-1+e^{\left(\theta_{\alpha}+\zeta\right) \bar{b}_{\alpha}}}{\theta_{\alpha}+\zeta}=\frac{2 a-\bar{b}_{\alpha}+\sqrt{\left(2 a-\bar{b}_{\alpha}\right)^{2}+8 a^{2}\left(\rho_{\beta}^{2}+2 \rho_{\beta}\right)}}{2\left(2+\rho_{\beta}\right)} \\
\bar{b}_{\beta}=\frac{\left(1+\rho_{\beta}\right)\left(\bar{b}_{\alpha}-2 a\right)+\sqrt{\left(2 a-\bar{b}_{\alpha}\right)^{2}+8 a^{2}\left(\rho_{\beta}^{2}+2 \rho_{\beta}\right)}}{2+\rho_{\beta}}
\end{array}\right.
$$

Figure 3 depicts the optimal strategic markups $b_{\alpha}$ and $b_{\beta}$ for $\zeta \in(0,0.1)$, where $a=1, \theta_{\alpha}=0.18$, and $\rho_{\beta}=0.85$. It shows that both optimal strategic markups are declining with $\zeta$, implying that as bidder $\alpha$ becomes more risk averse, both $b_{\alpha}$ and $b_{\beta}$ decrease (i.e., part (i) of Proposition 4 holds), $b_{\alpha}$ is more rapidly decreased than $b_{\beta}$, and the magnitude of the asymmetry effects on the optimal bid markup becomes smaller for bidder $\beta$ but becomes bigger for bidder $\alpha$. It also shows that the magnitude of the asymmetry effects is less for bidder $\beta$ than that for bidder $\alpha$ (i.e., part (ii) of Proposition 4 holds).

Example 6. Consider Example 4 again. Suppose that bidder $\alpha$ becomes more risk averse. Let $\rho_{\alpha}$ become $\rho_{\alpha}-\delta$, where $\delta$ is a positive real number. Then, the optimal strategic markups (22) become

$$
\begin{aligned}
& \bar{b}_{\alpha}=\frac{a\left(\rho_{\alpha}-\delta\right)(1+\bar{A})}{2+\rho_{\alpha}-\delta+\rho_{\beta}}, \\
& \bar{b}_{\beta}=\frac{a\left(\left(2+\rho_{\alpha}-\delta\right) \bar{A}-2-\left(\rho_{\alpha}-\delta\right)-2\left(\rho_{\beta}\right)\right)}{2+\rho_{\alpha}-\delta+\rho_{\beta}},
\end{aligned}
$$

where $\bar{A}=\sqrt{1+2 \rho_{\beta}\left(2+\rho_{\alpha}-\delta+\rho_{\beta}\right)}$.
Figure 4 depicts the optimal strategic markups $b_{\alpha}$ and $b_{\beta}$ for $\delta \in(0,0.69)$, where $a=1, \rho_{\alpha}=0.9$, and $\rho_{\beta}=0.2$. It shows that both optimal strategic markups are declining with $\delta$, implying that as bidder $\alpha$ becomes more risk averse, both $b_{\alpha}$ and $b_{\beta}$ decrease (i.e., part (i) of Proposition 3 holds), $b_{\alpha}$ is more rapidly decreased than $b_{\beta}$, and the magnitude of the asymmetry effects on the optimal bid markup becomes smaller for bidder $\beta$ but becomes bigger for bidder $\alpha$. It also shows that the magnitude of the asymmetry effects is more for bidder $\alpha$ than that for bidder $\beta$ (i.e., part (ii) of Proposition 3 holds).

From Proposition 3 and Proposition 4, we can find that the two bidders make uniformly higher bids as bidder $\beta$ or bidder $\alpha$ becomes more risk averse. This is consistent with the traditional result on symmetric auctions.

\subsection{Impact of the Degrees of Risk Aversion on Allocative Efficiency}

\section{Proposition 5}

(i) Suppose $v_{\beta}>v_{\alpha}$. Both auctions associated with Models $I$ and II are always efficient. 


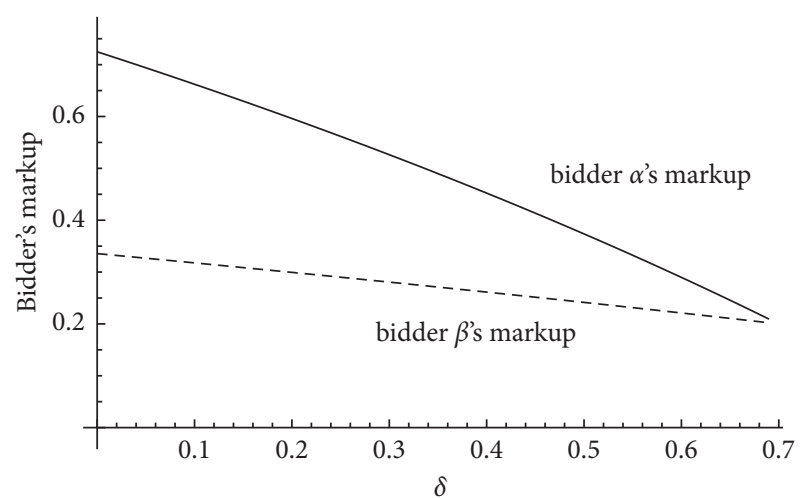

Figure 4: Plot of the optimal strategic markups $b_{\alpha}$ and $b_{\beta}$ increase with the degree of bidder $\alpha$ 's risk aversion.

(ii) Suppose $v_{\alpha}>v_{\beta}$. For Model I, the auction is inefficient if and only if $v_{\alpha}-v_{\beta}<2\left(a \rho_{\alpha}-b_{\beta}\right) /\left(2+\rho_{\alpha}\right)$; the auction is efficient if and only if $v_{\alpha}-v_{\beta}>2\left(a \rho_{\alpha}-b_{\beta}\right) /$ $\left(2+\rho_{\alpha}\right)$. For Model II, the auction is inefficient if and only if $v_{\alpha}-v_{\beta}<p\left(b_{\alpha}\right)$; the auction is efficient if and only if $v_{\alpha}-v_{\beta}>p\left(b_{\alpha}\right)$, where

$$
p\left(b_{\alpha}\right)=\frac{1}{2+\rho_{\beta}}\left(b_{\alpha}+2 a\left(1+\rho_{\beta}\right)-\sqrt{\left(2 a-b_{\alpha}\right)^{2}+8 a^{2}\left(\rho_{\beta}^{2}+2 \rho_{\beta}\right)}\right) .
$$

Proof. See Appendix.

Notice that if $v_{\beta}>v_{\alpha}$, the auctions are always efficient. In the following, we just consider that $v_{\alpha}>v_{\beta}$.

\section{Proposition 6}

(i) Consider Model I; suppose that bidder $\alpha$ 's risk aversion level remains unchanged. (a) If the auction is inefficient, as bidder $\beta$ becomes more risk averse, the more inefficient the allocation can be, while as bidder $\beta$ becomes less risk averse, allocative efficiency becomes ambiguous. (b) If the auction is efficient, as bidder $\beta$ becomes less risk averse, allocative efficiency becomes ambiguous, while as bidder $\beta$ becomes less risk averse, the auction is still efficient.

(ii) Consider Model II; suppose that bidder $\beta$ 's risk aversion level remains unchanged. (a) If the auction is inefficient, as bidder $\alpha$ becomes more risk averse, allocative efficiency becomes ambiguous. Consider Example 2. Suppose that $v_{\alpha}-v_{\beta}=0.02$ and $a=1$. Substituting $a=1$ and the related data in column 2 of Table 2 (i.e., $\theta_{\alpha}=0.3, \rho_{\beta}=0.8$, and $b_{\alpha}=0.8635$ ) into (26), we have $p\left(b_{\alpha}\right) \approx 0.0260$. Thus, $v_{\alpha}-v_{\beta}<p\left(b_{\alpha}\right)$, implying that the auction is inefficient by Proposition 5 (ii). Now, suppose that bidder $\alpha$ becomes more risk averse such that $\theta_{\alpha}=0.35$ or $\theta_{\alpha}=0.41$. For $\theta_{\alpha}=0.35$, using the data of column 3 in Table 2, we have $v_{\alpha}-v_{\beta}$ $<p\left(b_{\alpha}\right) \approx 0.0223$, implying that the auction is inefficient. But for $\theta_{\alpha}=0.41$, using the data of column 5 in Table 2, we have $v_{\alpha}-v_{\beta}>p\left(b_{\alpha}\right) \approx 0.0149$, implying that the auction is efficient by Proposition 5 (ii), while as bidder $\alpha$ becomes less risk averse, the auction becomes more inefficient. (b) If the auction is efficient, as bidder a becomes more risk averse, the auction is still efficient, while as bidder $\alpha$ becomes less risk averse, allocative efficiency becomes ambiguous.

Proof. See Appendix.

The following Examples 7 and 8 illustrate the magnitude of the asymmetry effects on the efficiency established in Proposition 6.

Example 7. Consider Example 1 again. Suppose that $v_{\alpha}>v_{\beta}$ in Proposition 6 (i). Then, the auction is efficient if $v_{\alpha}-b_{\alpha}>v_{\beta}-b_{\beta}$ (i.e., $v_{\alpha}-v_{\beta}>b_{\alpha}-b_{\beta}$ ).

Let $\theta_{\beta}$ become $\theta_{\beta}+\varepsilon$, implying that bidder $\beta$ becomes less risk averse and let $\theta_{\beta}$ become $\theta_{\beta}-\varepsilon$, implying that bidder $\beta$ becomes more risk averse, where $\varepsilon \in(0,0.06)$ :

(a) If the auction is inefficient. Let $v_{\alpha}-v_{\beta}=0.0025$, $\theta_{\beta}=0.6, \rho_{\alpha}=0.8$ and $a=1$, we have $b_{\alpha}-b_{\beta}=0.0071$. Then, $v_{\alpha}-v_{\beta}<b_{\alpha}-b_{\beta}$. Figure 5 shows the relationship between $b_{\alpha}-b_{\beta}$ and $v_{\alpha}-v_{\beta}$ as bidder $\beta$ becomes less or more risk averse. The $b_{\alpha}-b_{\beta}$ is found to be increasing as $\beta$ becomes more risk averse. And the higher the $b_{\alpha}-b_{\beta}$ is, the more inefficient the allocation can be. While as $\beta$ becomes less risk averse, $b_{\alpha}-b_{\beta}$ declines to be lower than $v_{\alpha}-v_{\beta}$, the auction may be efficient.

(b) If the auction is efficient. Let $v_{\alpha}-v_{\beta}=0.0117$, $\theta_{\beta}=0.6, \rho_{\alpha}=0.8$ and $a=1$, we have $b_{\alpha}-b_{\beta}=0.0071$. Then $v_{\alpha}-v_{\beta}>b_{\alpha}-b_{\beta}$. Figure 6 shows the relationship between $b_{\alpha}-b_{\beta}$ and $v_{\alpha}-v_{\beta}$ as bidder ${ }_{\beta}$ becomes less or more risk averse. Clearly, as $\beta$ becomes less risk averse $b_{\alpha}-b_{\beta}$ is always lower than $v_{\alpha}-v_{\beta}$, then, the auction is still efficient. While as $\beta$ becomes more risk averse, $b_{\alpha}-b_{\beta}$ may be higher than $v_{\alpha}-v_{\beta}$, then, the auction may become inefficient.

Example 8. Consider Example 2 again. Suppose that $v_{\alpha}>v_{\beta}$ in Proposition 6 (ii). Then, the auction is efficient if $v_{\alpha}-b_{\alpha}>v_{\beta}-b_{\beta}$, i.e., $v_{\alpha}-v_{\beta}>b_{\alpha}-b_{\beta}$.

Let $\theta_{\alpha}$ becomes $\theta_{\alpha}+\varepsilon$ implies that bidder $\beta$ becomes less risk averse and let $\theta_{\alpha}$ becomes $\theta_{\alpha}-\varepsilon$ implies that bidder $\alpha$ becomes more risk averse, where $\varepsilon \in(0,0.1)$ :

(a) Consider the auction when inefficient. Let $v_{\alpha}-v_{\beta}=0.020, \theta_{\alpha}=0.18, \rho_{\beta}=0.85$, and $a=1$; we have $b_{\alpha}-b_{\beta}=0.026$. Then, $v_{\alpha}-v_{\beta}<b_{\alpha}-b_{\beta}$. Figure 7 


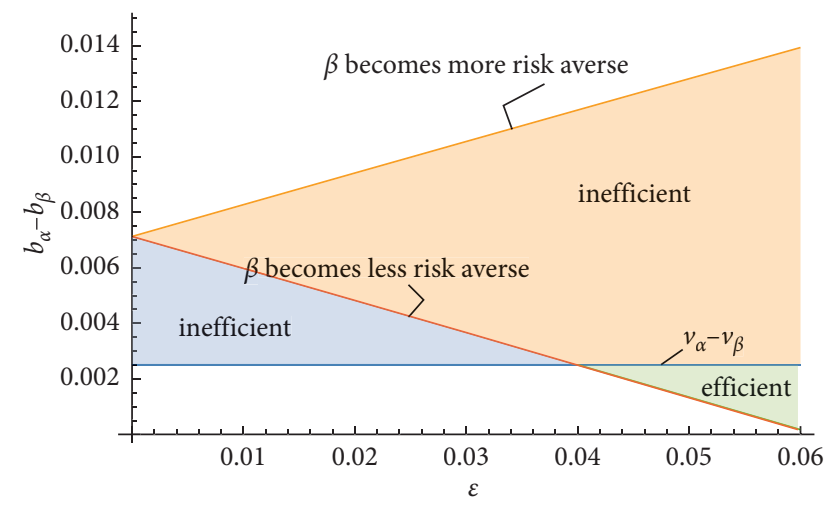

FIgURE 5: Plot showing that allocative efficiency is affected by the decrease of the degree of bidder $\beta$ 's risk aversion.

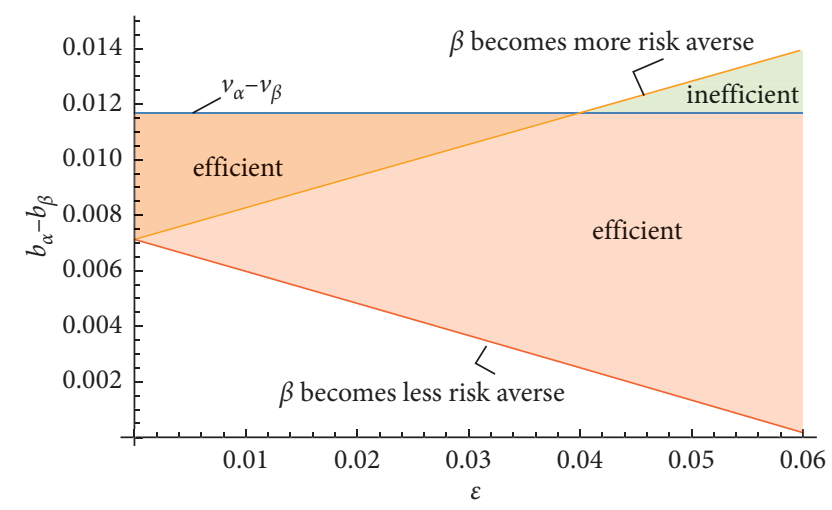

FIGURE 6: Plot showing that allocative efficiency is affected by the increase of the degree of bidder $\beta$ 's risk aversion.

depicts the relationship between $b_{\alpha}-b_{\beta}$ and $v_{\alpha}-v_{\beta}$ as bidder $\alpha$ becomes less or more risk averse. Clearly, $b_{\alpha}-b_{\beta}$ is increasing as $\alpha$ becomes less risk averse. And the higher the $b_{\alpha}-b_{\beta}$ is, the more inefficient the allocation can be. While as $\alpha$ becomes more risk

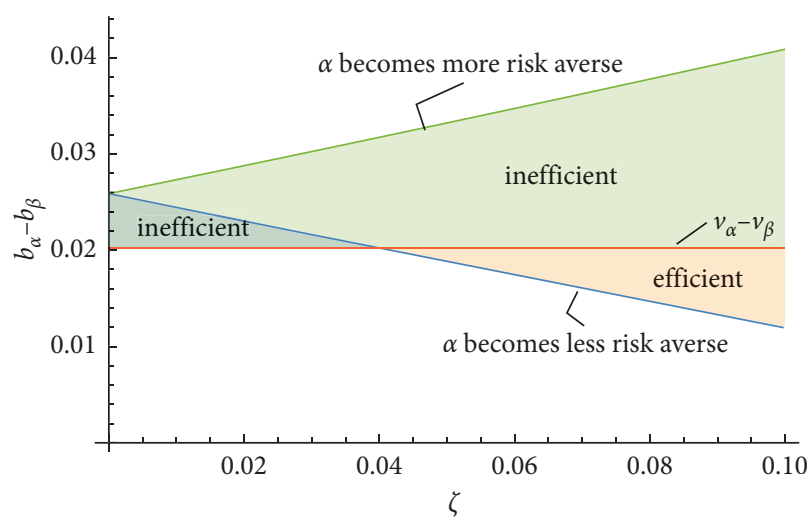

FIgURe 7: Plot shows that allocative efficiency is affected by the increase of the degree of bidder $\alpha$ 's risk aversion.

averse, $b_{\alpha}-b_{\beta}$ declines to be lower than $v_{\alpha}-v_{\beta}$; the auction may become efficient.

(b) Consider the auction when efficient. Let $v_{\alpha}-v_{\beta}=0.030, \theta_{\alpha}=0.18, \rho_{\beta}=0.85$, and $a=1$; we have $b_{\alpha}-b_{\beta}=0.026$. Then, $v_{\alpha}-v_{\beta}>b_{\alpha}-b_{\beta}$. Figure 8 shows that $b_{\alpha}-b_{\beta}$ is increasing as $\alpha$ becomes more risk averse and as $\alpha$ becomes less risk averse, $b_{\alpha}-b_{\beta}$ increases to be higher than $v_{\alpha}-v_{\beta}$, and the auction may become efficient.

\subsection{Impact of the Degrees of Risk Aversion on Expected} Revenue. Our following proposition shows that the seller's expected revenue increases with each bidder's risk aversion, which is the same as that in the standard first-price sealedbid auctions (see Riley and Samuelson [6], among others). This result for the standard first-price auctions comes directly from the increase of a bidder's equilibrium bid in risk aversion. However, this result in this paper cannot be obtained in the same way. The reason is as follows.

Under the assumption $b_{\alpha} \geq b_{\beta}$, the seller's expected revenue can be derived as (equation (10) of MM)

$$
\begin{aligned}
E R= & \int_{b_{\alpha}-b_{\beta}+\mu-a}^{a+\mu}\left(v_{\alpha}-b_{\alpha}\right) F\left(v_{\alpha}-b_{\alpha}+b_{\beta}\right) f\left(v_{\alpha}\right) \mathrm{d} v_{\alpha} \\
& +\int_{\mu-a}^{a-b_{\alpha}+b_{\beta}+\mu}\left(v_{\beta}-b_{\beta}\right) F\left(v_{\beta}+b_{\alpha}-b_{\beta}\right) f\left(v_{\beta}\right) \mathrm{d} v_{\beta}+\int_{a-b_{\alpha}+b_{\beta}+\mu}^{a+\mu}\left(v_{\beta}-b_{\beta}\right) f\left(v_{\beta}\right) \mathrm{d} v_{\beta} .
\end{aligned}
$$

By simplifying it, we have

$$
\mathrm{ER}=\frac{1}{4 a^{2}}\left(\frac{4}{3} a^{3}+4 \mu a^{2}-2 a^{2}\left(b_{\alpha}+b_{\beta}\right)+a\left(b_{\alpha}-b_{\beta}\right)^{2}-\frac{1}{6}\left(b_{\alpha}-b_{\beta}\right)^{3}\right) .
$$

\section{Proposition 7}

(i) In Model I, given that bidder $\alpha$ 's risk aversion level remains unchanged, the seller's expected revenue increases as bidder $\beta$ becomes more risk averse. (ii) In Model II, given that bidder $\beta$ 's risk aversion level remains unchanged, the seller's expected revenue increases as bidder $\alpha$ becomes more risk averse.

By Propositions 3 and 4, for Model I, the optimal bid markups of both bidders $\alpha$ and $\beta$ become smaller (i.e., both bidders bid higher) as bidder $\beta$ becomes more risk averse and the risk aversion level of bidder $\alpha$ is fixed, similarly, for Model II, the optimal strategic markups for both bidders become smaller (i.e., both bidders bid higher) as bidder $\alpha$ becomes more risk averse and the risk aversion level of 


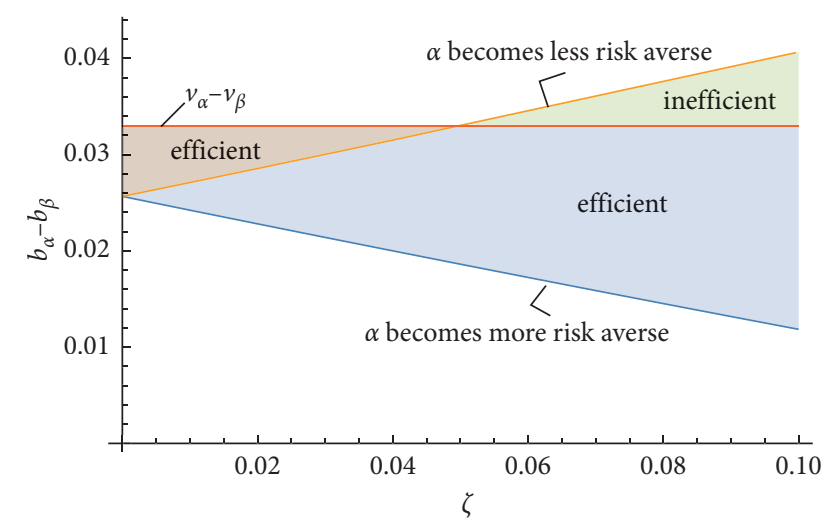

Figure 8: Plot showing that allocative efficiency is affected by the decrease of the degree of bidder $\alpha$ 's risk aversion.

bidder $\beta$ is fixed. Intuitively, this implies that the seller's expected revenue will become higher. Clearly, this result is not obvious by (28) (see the proof of this proposition for details).

Examples 9 and 10 illustrate the magnitude of the asymmetry effects on the seller's expected revenue established in Proposition 7.

Example 9. Consider Example 3 again. Suppose that bidder $\beta$ becomes more risk averse in Proposition 7 (i). Let $\theta_{\beta}$ becomes $\theta_{\beta}+\varepsilon$, where $\varepsilon$ is a positive real number. Then, the optimal strategic markups satisfy (21). Obviously, we cannot derive the closed form of the optimal strategic markups for both bidders. Then, we calculate it with numerical method. Substituting the numerical results obtained into (28), the seller's expected revenue is

$$
\widehat{E}_{R}=\frac{1}{4 a^{2}}\left(\frac{4}{3} a^{3}+4 \mu a^{2}-2 a^{2}\left(\widehat{b}_{\alpha}+\widehat{b}_{\beta}\right)+a\left(\widehat{b}_{\alpha}-\widehat{b}_{\beta}\right)^{2}-\frac{1}{6}\left(\widehat{b}_{\alpha}-\widehat{b}_{\beta}\right)^{3}\right) .
$$

In addition, in order to satisfy the optimal strategic markups for both bidders exist in the interval $\left(0, a \rho_{\alpha}\right]$, the range of parameter $\varepsilon$ we set is very small. Therefore, the nonlinear curve looks nearly linear in the following figure.

Figure 9 depicts the seller's expected revenue ER for $\varepsilon \in(0,0.1)$, where $a=1, \theta_{\beta}=0.6$ and $\rho_{\alpha}=0.8$. It shows that the seller's expected revenue is increasing with $\varepsilon$, implying that as bidder $\beta$ becomes more risk averse, the seller's expected revenue increases (i.e., part (i) of Proposition 7 holds).

Example 10. Consider Example 4 again. Suppose that bidder $\beta$ becomes more risk averse. Let $\rho_{\beta}$ become $\rho_{\beta}-\delta$, where $\delta$ is a positive real number. Then, the optimal strategic markups satisfy (23). By substituting (23) into (28), we obtain the seller's expected revenue (29).

Figure 10 depicts the seller's expected revenue $\widehat{E}_{R}$ for $\delta \in(0,0.55)$, where $a=1, \mu=5, \rho_{\alpha}=0.9$, and $\rho_{\beta}=0.6$. It shows that the seller's expected revenue is increasing with $\delta$, implying that as bidder $\beta$ becomes more risk averse, the seller's expected revenue increases (i.e., part (i) of Proposition 7 holds).

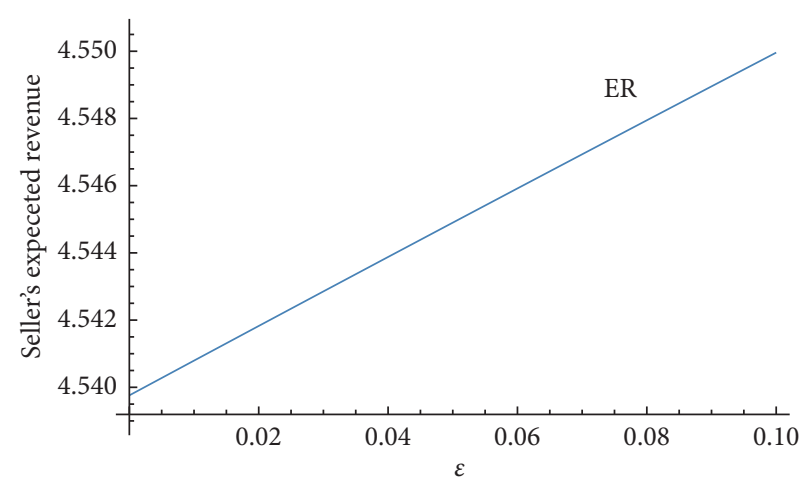

FIGURE 9: Plot of the seller's expected revenue ER increases with the degree of bidder $\beta$ 's risk aversion.

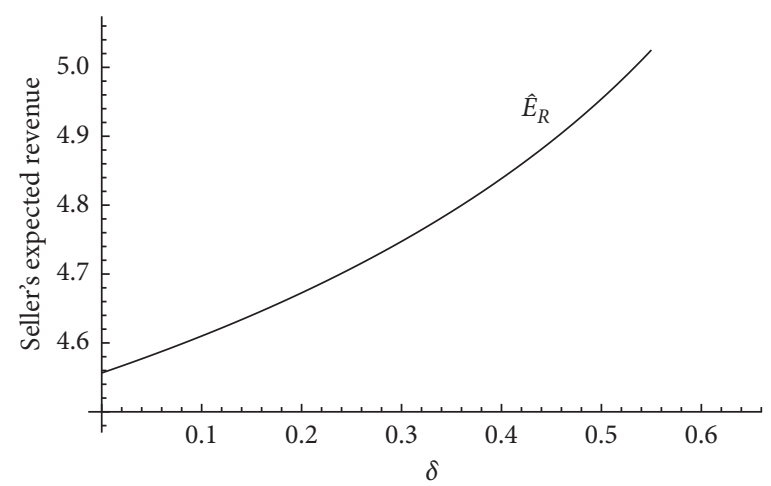

Figure 10: Plot of the seller's expected revenue increases with the degree of bidder $\beta$ 's risk aversion.

Example 11. Consider Example 5 again. Suppose that bidder $\alpha$ becomes more risk averse in Proposition 7 (ii). Let $\theta_{\alpha}$ become $\theta_{\alpha}+\zeta$, where $\zeta$ is a positive real number, and the larger $\zeta$ is, bidder $\alpha$ becomes more risk averse. Then, the optimal strategic markups satisfy (24). We cannot derive the closed form of the optimal strategic markups for both bidders. Then, we calculate it with numerical method. Substituting the numerical results obtained into (28), we obtain the seller's expected revenue

$$
\bar{E}_{R}=\frac{1}{4 a^{2}}\left(\frac{4}{3} a^{3}+4 \mu a^{2}-2 a^{2}\left(\bar{b}_{\alpha}+\bar{b}_{\beta}\right)+a\left(\bar{b}_{\alpha}-\bar{b}_{\beta}\right)^{2}-\frac{1}{6}\left(\bar{b}_{\alpha}-\bar{b}_{\beta}\right)^{3}\right) .
$$

Figure 11 depicts the seller's expected revenue $E R$ for $\zeta \in(0,0.1)$, where $a=1, \mu=5, \theta_{\alpha}=0.18$, and $\rho_{\beta}=0.85$. It is clear that ER increases with $\zeta$. It shows that the seller's expected revenue is increasing with $\zeta$, implying that as bidder $\alpha$ becomes more risk averse, the seller's expected revenue increases (i.e., part (ii) of Proposition 7 holds).

Example 12. Consider Example 6 again. Suppose that bidder $\alpha$ becomes more risk averse. Let $\rho_{\alpha}$ become $\rho_{\alpha}-\delta$, where $\delta$ is a positive real number. Then, the optimal strategic markups satisfy (25). By substituting (25) into (28), we obtain the seller's expected revenue (30).

Figure 12 depicts the seller's expected revenue $\bar{E}_{R}$ for $\delta \in(0,0.69)$, where $a=1, \mu=5, \rho_{\alpha}=0.9$, and $\rho_{\beta}=0.2$. It shows 


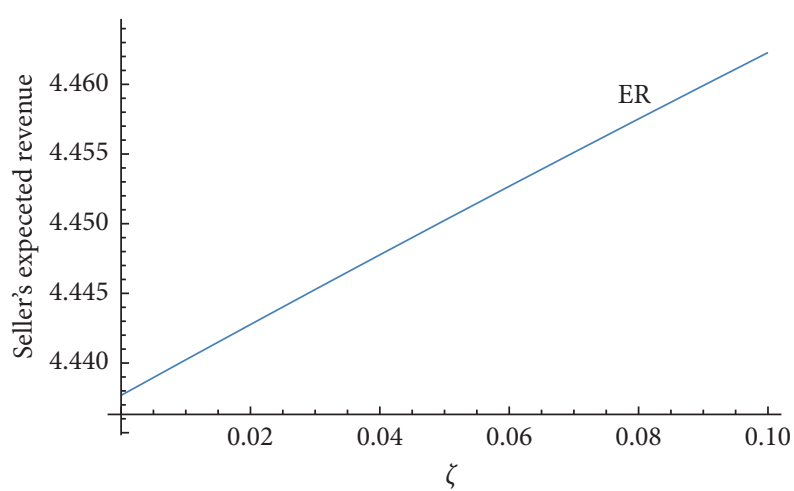

FIGURE 11: Plot of the seller's expected revenue ER increases with the degree of bidder $\alpha$ 's risk aversion.

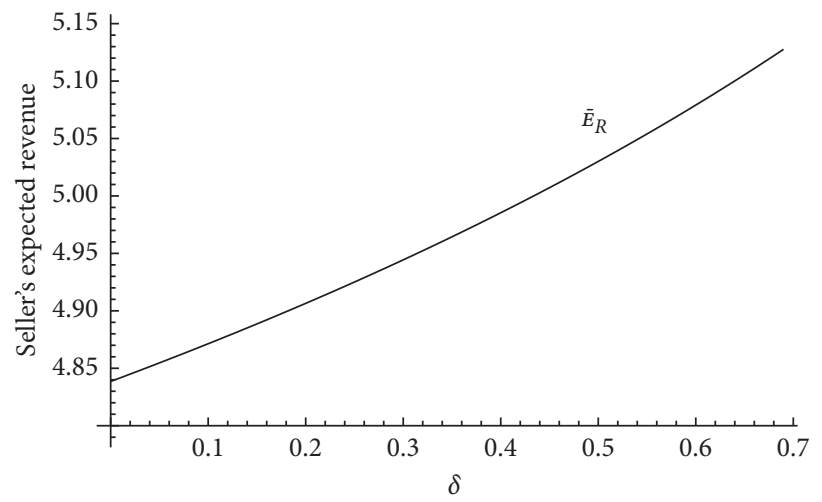

FIgURE 12: Plot of the seller's expected revenue increases with the degree of bidder $\alpha$ 's risk aversion.

that the seller's expected revenue is increasing with $\delta$, implying that as bidder $\alpha$ becomes more risk averse, the seller's expected revenue increases (i.e., part (ii) of Proposition 7 holds).

\section{Conclusion}

In this paper, we study the asymmetric first-price sealedbid auctions with two bidders where one bidder has a general concave utility function and the other one has a CRRA utility function. We first establish the existence and uniqueness of the optimal strategic markups of both bidders (then the existence and uniqueness of the asymmetric equilibrium bidding strategies are proved). Then, analyze the impact of one bidder's risk aversion on both bidders' optimal markups, allocative efficiency of the auction, and the seller's expected revenue. We have shown that when one bidder becomes more risk averse and his opponent's risk aversion level is fixed, (i) both bidders will reduce their markups; (ii) the change in his markup is greater than the change in his opponent's markup; and (iii) the seller's expected revenue will increase. In addition, the change of one bidder's risk aversion level can also add complexity to the allocative efficiency.

\section{Appendix}

Proof of Proposition 1.

Part (i) The first-order condition of bidder $\alpha$ 's expected utility maximization problem (6) is

$$
b_{\alpha}=-\frac{\rho_{\alpha} P\left(b_{\alpha}, b_{\beta}\right)}{P_{1}^{\prime}\left(b_{\alpha}, b_{\beta}\right)} .
$$

Substituting (8) into (A.1) and simplifying, the authors obtain (14). The first-order condition of bidder $\beta$ 's expected utility maximization problem (7) is

$$
\gamma_{\beta}\left(b_{\beta}\right)=\frac{u_{\beta}\left(b_{\beta}\right)}{u_{\beta}^{\prime}\left(b_{\beta}\right)}=-\frac{Q\left(b_{\alpha}, b_{\beta}\right)}{Q_{2}^{\prime}\left(b_{\alpha}, b_{\beta}\right)} .
$$

Substituting (9) into (A.2) and simplifying, the authors obtain

$$
\gamma_{\beta}\left(b_{\beta}\right)=\frac{4 a^{2}+4 a\left(b_{\alpha}-b_{\beta}\right)-\left(b_{\alpha}-b_{\beta}\right)^{2}}{2\left(2 a+b_{\beta}-b_{\alpha}\right)} .
$$

By substituting (14) into (A.3) and simplifying, the authors obtain (15). (ii) Differentiating (16) with respect to $x$, the authors obtain 


$$
G^{\prime}(x)=\frac{-2 a^{2}\left(\rho_{\alpha}+2\right)-(x+2 a)^{2}}{(x+2 a)^{2}\left(\rho_{\alpha}+2\right)}<0 .
$$

Define $F(x)=\gamma_{\beta}(x)-G(x)$ on $[0, \infty)$. Then, $F(0)=\gamma_{\beta}$ $(0)-G(0)=-G(0)<0$ since $G(0)=a\left(\left(\rho_{\alpha}+2\right)^{2}-2\right) / \rho_{\alpha}+$ $2>0$. The concavity of $u_{\beta}$ implies that $\gamma_{\beta}(x) \geq x$. Thus,

$$
F(x) \geq x-G(x)=H(x),
$$

where $H(x)=x-\left(\left(2 a^{2}\left(2+\rho_{\alpha}\right) / x+2 a\right)-\left(x+2 a / 2+\rho_{\alpha}\right)\right)$. Clearly, $H^{\prime}(x)>0$ for $x \in[0, \infty)$. Solving $H(x)>0$, or equivalently, $\left(3+\rho_{\alpha}\right) x^{2}+a\left(8+2 \rho_{\alpha}\right) x-a^{2}\left(4+2 \rho_{\alpha}^{2}+8 \rho_{\alpha}\right)$ $>0$, we have $x>x_{1}$, where $H\left(x_{1}\right)=0$,

$$
\begin{aligned}
x_{1}= & \frac{a}{2\left(3+\rho_{\alpha}\right)}\left(-\left(8+2 \rho_{\alpha}\right)+\sqrt{\left(8+2 \rho_{\alpha}\right)^{2}+4\left(3+\rho_{\alpha}\right)\left(4+2 \rho_{\alpha}^{2}+8 \rho_{\alpha}\right)}\right), \\
= & \frac{a}{\left(3+\rho_{\alpha}\right)}\left(-\left(4+\rho_{\alpha}\right)+\sqrt{\left(4+\rho_{\alpha}\right)^{2}+\left(3+\rho_{\alpha}\right)\left(4+2 \rho_{\alpha}^{2}+8 \rho_{\alpha}\right)}\right) \\
& >\frac{a}{\left(3+\rho_{\alpha}\right)}\left(-\left(4+\rho_{\alpha}\right)+\sqrt{\left(4+\rho_{\alpha}\right)^{2}+\rho_{\alpha}^{2}\left(3+\rho_{\alpha}\right)^{2}+2 \rho_{\alpha}\left(3+\rho_{\alpha}\right)\left(4+\rho_{\alpha}\right)}\right) \\
= & \frac{a}{\left(3+\rho_{\alpha}\right)}\left(-\left(4+\rho_{\alpha}\right)+\sqrt{\left(4+\rho_{\alpha}+\rho_{\alpha}\left(3+\rho_{\alpha}\right)\right)^{2}}\right) \\
= & \frac{a}{\left(3+\rho_{\alpha}\right)}\left(\rho_{\alpha}\left(3+\rho_{\alpha}\right)\right) \\
= & a \rho_{\alpha} .
\end{aligned}
$$

Let $x_{2}>x_{1}$; then, $H\left(x_{2}\right)>H\left(x_{1}\right)=0$ since $H(x)$ is increasing. Thus, using (A.4), the authors have $F\left(x_{2}\right)>0$. By the intermediate value theorem, there is a $b_{\beta}^{*} \in\left(0, x_{2}\right)$ such that $F\left(b_{\beta}^{*}\right)=0$. Combining the assumption of $b_{\alpha} \geq b_{\beta}$ with (14), the authors have $b_{\beta} \leq a \rho_{\alpha}$. Since $x_{2}>x_{1}>a \rho_{\alpha}$, there exists bidder $\beta$ 's optimal markup with $b_{\beta}^{*} \in\left(0, a \rho_{\alpha}\right]$. It follows that bidder $\alpha$ 's optimal markup $b_{\alpha}^{*}$ also exists because of (14). Since $\gamma_{\beta}^{\prime}(x)>0$ and $G^{\prime}(x)<0$ for $x \geq 0, F^{\prime}(x)>0$ for $x \geq 0$. Thus, $b_{\beta}^{*}$ is unique. It follows from (14) that $b_{\alpha}^{*}$ is also unique.
Furthermore, the authors have $0<b_{\alpha}^{*} \leq \rho_{\alpha} \quad\left(2 a+a \rho_{\alpha}\right)$ / $\left(2+\rho_{\alpha}\right)=a \rho_{\alpha}$ by (14). The authors need to verify $b_{\alpha}^{*} \geq b_{\beta}^{*}$. In fact, using (14) and $a \rho_{\alpha} \geq b_{\beta}^{*}$, the authors have $b_{\alpha}^{*} \geq\left(2 b_{\beta}^{*}+b_{\beta}^{*} \rho_{\alpha}\right) /\left(2+\rho_{\alpha}\right)=b_{\beta}^{*}$.

Part (iii) Define $F\left(a, \rho_{\alpha} ; b_{\beta}\right)=\gamma_{\beta}\left(b_{\beta}\right)-G\left(b_{\beta}\right)$. Then, by (15) and part (ii) of Proposition 1, the optimal markup $b_{\beta}^{*}$ over $\left(0, a \rho_{\alpha}\right]$ satisfies $F\left(a, \rho_{\alpha} ; b_{\beta}\right)=0$. Since $\gamma_{i}(x)>0$ and $G^{\prime}$ $(x)<0$ for $x \geq 0, F_{b_{\beta}}^{\prime}\left(a, \rho_{\alpha} ; b_{\beta}\right)=\gamma_{\alpha}^{\prime}\left(b_{\beta}\right)-G^{\prime}\left(b_{\beta}\right)>0$. Since $b_{\beta} \in\left(0, a \rho_{\alpha}\right]$, the authors have

$$
\begin{aligned}
\frac{\partial F\left(a, \rho_{\alpha} ; b_{\beta}\right)}{\partial a}= & \frac{-4 a\left(\rho_{\alpha}+2\right)^{2}\left(b_{\beta}+2 a\right)+4 a^{2}\left(\rho_{\alpha}+2\right)+2\left(b_{\beta}+2 a\right)^{2}}{\left(b_{\beta}+2 a\right)^{2}\left(\rho_{\alpha}+2\right)} \\
= & \frac{-4 a\left(\rho_{\alpha}+2\right)^{2} b_{\beta}-4 a^{2}\left(\rho_{\alpha}+2\right)+2\left(b_{\beta}+2 a\right)^{2}}{\left(b_{\beta}+2 a\right)^{2}\left(\rho_{\alpha}+2\right)} \\
= & \frac{-4 a\left(\rho_{\alpha}+2\right)^{2} b_{\beta}+2 b_{\beta}^{2}-4 a^{2} \rho_{\alpha}+8 a b_{\beta}}{\left(b_{\beta}+2 a\right)^{2}\left(\rho_{\alpha}+2\right)} \\
& <\frac{\left(-4 a\left(\rho_{\alpha}+2\right)^{2}+8 a\right) b_{\beta}+2\left(a \rho_{\alpha}\right)^{2}-4 a^{2} \rho_{\alpha}}{\left(b_{\beta}+2 a\right)^{2}\left(\rho_{\alpha}+2\right)} \\
= & \frac{\left(-4 a\left(\rho_{\alpha}+2\right)^{2}+8 a\right) b_{\beta}+4 a^{2}\left(\rho_{\alpha}^{2}-1\right)}{\left(b_{\beta}+2 a\right)^{2}\left(\rho_{\alpha}+2\right)}
\end{aligned}
$$


Thus,

$$
\frac{\partial b_{\beta}\left(a, \rho_{\alpha}\right)}{\partial a}=-\frac{\left(\partial F\left(a, \rho_{\alpha} ; b_{\beta}\right) / \partial a\right)}{\partial F\left(a, \rho_{\alpha} ; b_{\beta}\right) / \partial b_{\beta}}>0 .
$$

Then, $b_{\beta}$ is increasing with $a$. It follows by (14) that $b_{\alpha}$ is also increasing with $a$.

\section{Proof of Proposition 2.}

(i) The first-order condition of bidder $\beta$ 's expected utility maximization problem (11) is

$$
b_{\beta}=-\frac{\rho_{\beta} Q\left(b_{\alpha}, b_{\beta}\right)}{Q_{2}^{\prime}\left(b_{\alpha}, b_{\beta}\right)} .
$$

Similarly, the first-order condition of bidder $\alpha$ 's expected utility maximization problem (10) is

$$
\gamma_{\alpha}\left(b_{\alpha}\right)=\frac{u_{\alpha}\left(b_{\alpha}\right)}{u_{\alpha}^{\prime}\left(b_{\alpha}\right)}=-\frac{P\left(b_{\alpha}, b_{\beta}\right)}{P_{1}^{\prime}\left(b_{\alpha}, b_{\beta}\right)} .
$$

Substituting (8) into (A.10) and simplifying, the authors obtain

$$
\gamma_{\alpha}\left(b_{\alpha}\right)=\frac{2 a-\left(b_{\alpha}-b_{\beta}\right)}{2}
$$

Substituting (9) into (A.9) and simplifying, the authors obtain

$$
b_{\beta}=-\rho_{\beta} \frac{\left[4 a^{2}+4 a\left(b_{\alpha}-b_{\beta}\right)-\left(b_{\alpha}-b_{\beta}\right)^{2}\right]}{2\left(b_{\alpha}-b_{\beta}-2 a\right)} .
$$

After simplifying, the authors obtain a quadratic equation with the unknown variable of $b_{\beta}$ :

$$
\left(2+\rho_{\beta}\right) b_{\beta}^{2}+2\left(1+\rho_{\beta}\right)\left(2 a-b_{\alpha}\right) b_{\beta}+\left(b_{\alpha}^{2}-4 a^{2}-4 a b_{\alpha}\right) \rho_{\beta}=0 .
$$

Solving it for $b_{\beta}$, the authors obtain two solutions:

$$
\begin{aligned}
& b_{\beta}^{1}=\frac{\left(1+\rho_{\beta}\right)\left(b_{\alpha}-2 a\right)+\sqrt{\Delta}}{\left(2+\rho_{\beta}\right)}=g\left(b_{\alpha}\right), \\
& b_{\beta}^{2}=\frac{\left(1+\rho_{\beta}\right)\left(b_{\alpha}-2 a\right)-\sqrt{\Delta}}{\left(2+\rho_{\beta}\right)},
\end{aligned}
$$

where

$$
\begin{aligned}
\Delta & =\left(1+\rho_{\beta}\right)^{2}\left(2 a-b_{\alpha}\right)^{2}-\left(2+\rho_{\beta}\right) \rho_{\beta}\left(b_{\alpha}^{2}-4 a^{2}-4 a b_{\alpha}\right), \\
& =\left(2 a-b_{\alpha}\right)^{2}+8 a^{2}\left(\rho_{\beta}^{2}+2 \rho_{\beta}\right)>0 .
\end{aligned}
$$

Furthermore, the concavity of $u_{\alpha}$ implies that $\gamma_{\alpha}\left(b_{\alpha}\right) \geq b_{\alpha}$ for $b_{\alpha} \geq 0$. Thus, using (A.11), the authors have $b_{\alpha} \leq\left[2 a-\left(b_{\alpha}-b_{\beta}\right)\right] / 2$. This and $b_{\alpha} \geq b_{\beta}$ imply that $b_{\alpha} \leq a$. Since $b_{\alpha} \leq a, b_{\beta}^{2}<0$. The authors reject $b_{\beta}^{2}$ on economic grounds and get (17) after omitting the subscript 1. By substituting (17) into (A.11) and simplifying, the authors obtain (18).

Part (ii) Combining $b_{\alpha} \geq b_{\beta}$ with (17) and (19), the authors have

$$
b_{\alpha}-b_{\beta}=b_{\alpha}-g\left(b_{\alpha}\right)=\frac{1}{2+\rho_{\beta}}\left(b_{\alpha}+2 a\left(1+\rho_{\beta}\right)-\sqrt{\left(2 a-b_{\alpha}\right)^{2}+8 a^{2}\left(\rho_{\beta}^{2}+2 \rho_{\beta}\right)}\right) \geq 0 \text {. }
$$

Thus,

$$
\begin{aligned}
b_{\alpha}+2 a\left(1+\rho_{\beta}\right)-\sqrt{\left(2 a-b_{\alpha}\right)^{2}+8 a^{2}\left(\rho_{\beta}^{2}+2 \rho_{\beta}\right)} & \geq 0, \\
b_{\alpha}+2 a\left(1+\rho_{\beta}\right) & \geq \sqrt{\left(2 a-b_{\alpha}\right)^{2}+8 a^{2}\left(\rho_{\beta}^{2}+2 \rho_{\beta}\right),} \\
b_{\alpha}^{2}+4 a^{2}\left(1+\rho_{\beta}\right)^{2}+4 a\left(1+\rho_{\beta}\right) b_{\alpha} & \geq\left(2 a-b_{\alpha}\right)^{2}+8 a^{2}\left(\rho_{\beta}^{2}+2 \rho_{\beta}\right), \\
b_{\alpha}\left(2+\rho_{\beta}\right) & \geq a \rho_{\beta}\left(2+\rho_{\beta}\right) .
\end{aligned}
$$

Therefore, $b_{\alpha} \geq a \rho_{\beta}$. Then, using $b_{\alpha} \leq a$ from the proof of part (i), the authors have $b_{\alpha} \in\left[a \rho_{\beta}, a\right]$.

Differentiating (20) with respect to $x$, the authors obtain

$$
L^{\prime}(x)=\frac{-1+\left(b_{\alpha}-2 a / \sqrt{(2 a-x)^{2}+8 a^{2}\left(\rho_{\beta}^{2}+2 \rho_{\beta}\right)}\right)}{2\left(2+\rho_{\beta}\right)}<0 .
$$


Define $h\left(\rho_{\beta}\right)=1+\sqrt{1+8\left(\rho_{\beta}^{2}+2 \rho_{\beta}\right)} / 2\left(2+\rho_{\beta}\right)$ on $(0,1]$. Then, $L(a)=a h\left(\rho_{\beta}\right)$ by $(20)$. Since

$$
h^{\prime}\left(\rho_{\beta}\right)=\frac{30-2 \sqrt{1+8\left(\rho_{\beta}^{2}+2 \rho_{\beta}\right)}+16 \rho_{\beta}}{4\left(2+\rho_{\beta}\right)^{2} \sqrt{1+8\left(\rho_{\beta}^{2}+2 \rho_{\beta}\right)}}>0,
$$

$L(a)=a h\left(\rho_{\beta}\right) \leq a h(1)=a$.

Define $Q(x)=\gamma_{\alpha}(x)-L(x)$ on $[0, \infty)$. Then, $Q(0)=\gamma_{\alpha}$ (0) $-L(0)<0$ by $L(0)>0$. The concavity of $u_{\alpha}$ implies that $\gamma_{\alpha}$ $(x) \geq x$. Thus,

$$
\begin{aligned}
Q(a)= & \gamma_{\alpha}(a)-L(a), \\
\geq & a-L(a) \\
& =a-\frac{2 a-a+\sqrt{(2 a-a)^{2}+8 a^{2}\left(\rho_{\beta}^{2}+2 \rho_{\beta}\right)}}{2\left(2+\rho_{\beta}\right)} \\
& =\frac{a\left(3+2 \rho_{\beta}-\sqrt{8 \rho_{\beta}^{2}+16 \rho_{\beta}+1}\right)}{2\left(2+\rho_{\beta}\right)} \\
& =\frac{a\left(\left(3+2 \rho_{\beta}\right)^{2}-\left(\sqrt{8 \rho_{\beta}^{2}+16 \rho_{\beta}+1}\right)^{2}\right)}{2\left(2+\rho_{\beta}\right)\left(3+2 \rho_{\beta}+\sqrt{8 \rho_{\beta}^{2}+16 \rho_{\beta}+1}\right)} \\
& =\frac{a\left(8-4 \rho_{\beta}^{2}-4 \rho_{\beta}\right)}{2\left(2+\rho_{\beta}\right)\left(3+2 \rho_{\beta}+\sqrt{8 \rho_{\beta}^{2}+16 \rho_{\beta}+1}\right)}>0 .
\end{aligned}
$$

By the intermediate value theorem, there is a $b_{\alpha}^{*} \in(0, a)$ such that $Q\left(b_{\alpha}^{*}\right)=0$. Since the authors have proved that $b_{\alpha} \in\left[a \rho_{\beta}, a\right]$, there exists bidder $\alpha$ 's optimal markup with $b_{\alpha}^{*} \in\left[a \rho_{\beta}, a\right)$. It follows that bidder $\beta$ 's optimal markup $b_{\beta}^{*}$ also exists because of (17). Since $\gamma_{\alpha}^{\prime}(x)>0$ and $L^{\prime}(x)<0$ for $x \geq 0, Q^{\prime}(x)>0$ for $x \geq 0$. Thus, $b_{\alpha}^{*}$ is unique. It follows from (17) that $b_{\beta}^{*}$ is also unique.

Differentiating (19) with respect to $x$, the authors obtain

$$
g^{\prime}(x)=\frac{\left(1+\rho_{\beta}\right) \sqrt{(2 a-x)^{2}+8 a^{2}\left(\rho_{\beta}^{2}+2 \rho_{\beta}\right)}+(x-2 a)}{\left(2+\rho_{\beta}\right) \sqrt{(2 a-x)^{2}+8 a^{2}\left(\rho_{\beta}^{2}+2 \rho_{\beta}\right)}}>0,
$$

which implies that $g(x)$ is increasing on $\left[a \rho_{\beta}, a\right)$. It follows from $b_{\alpha}^{*} \in\left[a \rho_{\beta}, a\right)$ and (17) that we have

$$
\begin{aligned}
b_{\beta} \geq & g\left(a \rho_{\beta}\right)=\frac{1}{2+\rho_{\beta}}\left(\left(1+\rho_{\beta}\right)\left(a \rho_{\beta}-2 a\right)-\sqrt{\left(2 a-a \rho_{\beta}\right)^{2}+8 a^{2}\left(\rho_{\beta}^{2}+2 \rho_{\beta}\right)}\right)=\alpha \rho_{\beta}, \\
b_{\beta}<g(a)= & \frac{1}{2+\rho_{\beta}}\left(\left(1+\rho_{\beta}\right)(a-2 a)-\sqrt{(2 a-a)^{2}+8 a^{2}\left(\rho_{\beta}^{2}+2 \rho_{\beta}\right)}\right) \\
= & \frac{a}{2+\rho_{\beta}}\left(-\left(1+\rho_{\beta}\right)+\sqrt{1+8 \rho_{\beta}^{2}+16 \rho_{\beta}}\right) \\
& <\frac{a}{2+\rho_{\beta}}\left(-\left(1+\rho_{\beta}\right)+\sqrt{\left(3+2 \rho_{\beta}\right)^{2}}\right)=a .
\end{aligned}
$$

By (17), we need to verify $b_{\beta}^{*} \leq b_{\alpha}^{*}$. In fact, using (17) and $a \rho_{\beta} \leq b_{\alpha}^{*}$, we have

$$
b_{\beta}^{*} \leq \frac{1}{2+\rho_{\beta}}\left(\left(1+\rho_{\beta}\right) b_{\alpha}^{*}-2 a-2 b_{\alpha}^{*}-\sqrt{\left(2 a-b_{\alpha}^{*}\right)^{2}+8\left(b_{\alpha}^{*}\right)^{2}+16 a b_{\alpha}^{*}}\right)=b_{\alpha}^{*}
$$


Part (iii) Define $F\left(a, \rho_{\beta} ; b_{\alpha}\right)=\gamma_{\alpha}\left(b_{\alpha}\right)-L\left(b_{\alpha}\right)$. Then, by (18) and part (ii) of Proposition 2, the optimal markup $b_{\alpha}^{*}$ over $\left[a \rho_{\beta}, a\right)$ satisfies $F\left(a, \rho_{\beta} ; b_{\alpha}\right)=0$. Since $\gamma_{i}^{\prime}(x)>0$ and $L^{\prime}$ $(x)<0$ for $x \geq 0, F_{b_{\alpha}}^{\prime}\left(a, \rho_{\alpha} ; b_{\beta}\right)=\gamma_{\alpha}^{\prime}\left(b_{\alpha}\right)-L^{\prime}\left(b_{\alpha}\right)>0$. Since $b_{\alpha} \in\left[a \rho_{\beta}, a\right)$, the authors have

$$
\frac{\partial F\left(a, \rho_{\alpha} ; b_{\beta}\right)}{\partial a}=-\frac{2+\left(2\left(2 a-b_{\alpha}\right)+8 a\left(\rho_{\beta}^{2}+2 \rho_{\beta}\right)\right) \sqrt{\left(2 a-b_{\alpha}\right)^{2}+8 a^{2}\left(\rho_{\beta}^{2}+2 \rho_{\beta}\right)}}{2\left(2+\rho_{\beta}\right)}<0 .
$$

Thus,

$$
\frac{\partial b_{\alpha}\left(a, \rho_{\beta}\right)}{\partial a}=-\frac{\partial F\left(a, \rho_{\beta} ; b_{\alpha}\right) / \partial a}{\partial F\left(a, \rho_{\beta} ; b_{\alpha}\right) / \partial b_{\alpha}}>0 .
$$

Then, $b_{\alpha}$ is increasing with $a$. It follows by (17) that $b_{\beta}$ is also increasing with $a$.

\section{Proof of Proposition 3.}

Part (i) By Proposition 1 (i), the authors have

$$
\hat{\gamma}_{\beta}\left(\widehat{b}_{\beta}\right)=G\left(\widehat{b}_{\beta}\right), \quad \text { and } \gamma_{\beta}\left(b_{\beta}\right)=G\left(b_{\beta}\right) \text {. }
$$

The authors prove $\widehat{b}_{\beta}<b_{\beta}$ by contradiction. Suppose that $\widehat{b}_{\beta}>b_{\beta}$ or $\widehat{b}_{\beta}=b_{\beta}$. The authors first assume $\widehat{b}_{\beta}>b_{\beta}$. Since $R_{\beta}(\cdot)>R_{\beta}(\cdot)$ implies $\hat{\gamma}_{\beta}(\cdot)>\gamma_{\beta}(\cdot)$, the authors have $\hat{\gamma}_{\beta}\left(\widehat{b}_{\beta}\right)>\gamma\left(\widehat{b}_{\beta}\right)$. Since $\gamma_{\beta}(x)$ strictly increases in $x$, $\gamma_{\beta}\left(\vec{b}_{\beta}\right)>\gamma_{\beta}\left(b_{\beta}\right)$. Therefore, the authors have $\widehat{\gamma}_{\beta}\left(\widehat{b}_{\beta}\right)>\gamma\left(b_{\beta}\right)$. Since $G(x)$ is strictly decreasing in $x, G\left(b_{\beta}\right)<G\left(b_{\beta}\right)$. It follows from the first equality of (A.26), $\gamma_{\beta}\left(b_{\beta}\right)<G\left(b_{\beta}\right)$, which contradicts with the second equality of (A.26). Next, the authors assume $\widehat{b}_{\beta}=b_{\beta}$. Then, $\widehat{\gamma}_{\beta}\left(\widehat{b}_{\beta}\right)=\gamma_{\beta}\left(b_{\beta}\right)$ which contradicts with $\hat{\gamma}_{\beta}(\cdot)>\gamma_{\beta}(\cdot)$.

By Proposition 1 (i),

$$
\widehat{b}_{\alpha}=\frac{\rho_{\alpha}\left(2 a+\widehat{b}_{\beta}\right)}{2+\rho_{\alpha}} .
$$

Using (14), (A.27), and $\widehat{b}_{\beta}<b_{\beta}$, the authors have $\widehat{b}_{\alpha}<b_{\alpha}$. (ii) Using (14) and (A.27), $b_{\alpha}-\widehat{b}_{\alpha}=\rho_{\alpha} /\left(2+\rho_{\alpha}\right)\left(b_{\beta}-\widehat{b}_{\beta}\right)$. Thus, by $\rho_{\alpha} \in(0,1]$, the authors have $b_{\alpha}-\widehat{b}_{\alpha}<b_{\beta}-b_{\beta}$.

Proof of Proposition 4.

(i) By Proposition $2(\mathrm{i}), b_{\beta}=g\left(b_{\alpha}\right)$ and $\gamma_{\alpha}\left(b_{\alpha}\right)=L\left(b_{\alpha}\right)$, $\bar{b}_{\beta}=g\left(\bar{b}_{\alpha}\right)$, and $\bar{\gamma}_{\alpha}=L\left(\bar{b}_{\alpha}\right)$. We can prove $\bar{b}_{\alpha}<b_{\alpha}$ in a similar way by which we prove $\widehat{b}_{\beta}<b_{\beta}$ in Proposition 3. Thus, $\bar{b}_{\beta}<b_{\beta}$ since $g(x)$ is increasing on $\left[a \rho_{\beta}, a\right)$.

(ii) Let $m=\left(2 a-b_{\alpha}\right)^{2}+8 a^{2}\left(\rho_{\beta}^{2}+2 \rho_{\beta}\right), n=\left(2 a-\bar{b}_{\alpha}\right)$ $2^{2}+8 a^{2}\left(\rho_{\beta}^{2}+2 \rho_{\beta}\right)$. Then, using (17), we obtain

$$
\begin{aligned}
b_{\beta}-\bar{b}_{\beta}= & \frac{\left(1+\rho_{\beta}\right)\left(b_{\alpha}-\bar{b}_{\alpha}\right)+\sqrt{m}-\sqrt{n}}{2+\rho_{\beta}}, \\
= & \frac{\left(1+\rho_{\beta}\right)\left(b_{\alpha}-\bar{b}_{\alpha}\right)+\left(\left(\left(2 a-b_{\alpha}\right)^{2}-\left(2 a-\bar{b}_{\alpha}\right)^{2}\right) / \sqrt{m}+\sqrt{n}\right)}{2+\rho_{\beta}} \\
= & \frac{\left(1+\rho_{\beta}\right)\left(b_{\alpha}-\bar{b}_{\alpha}\right)+\left(\left(b_{\alpha}-\bar{b}_{\alpha}\right)\left(b_{\alpha}+\bar{b}_{\alpha}-4 a\right)\right) / \sqrt{m}+\sqrt{n}}{2+\rho_{\beta}} \\
= & \left(\frac{1+\rho_{\beta}}{2+\rho_{\beta}}+\frac{b_{\alpha}+\bar{b}_{\alpha}-4 a}{\left(2+\rho_{\beta}\right)(\sqrt{m}+\sqrt{n})}\right)\left(b_{\alpha}-\bar{b}_{\alpha}\right) \\
& <\left(\frac{1+\rho_{\beta}}{2+\rho_{\beta}}+\frac{\bar{b}_{\alpha}+b_{\alpha}-4 a}{\left(2+\rho_{\beta}\right)\left(1+\rho_{\beta}\right)\left[4 a-\left(\bar{b}_{\alpha}+b_{\alpha}\right)\right]}\right)\left(b_{\alpha}-\bar{b}_{\alpha}\right) \\
& <\left(\frac{1+\rho_{\beta}}{2+\rho_{\beta}}-\frac{1}{\left(2+\rho_{\beta}\right)\left(1+\rho_{\beta}\right)}\right)\left(b_{\alpha}-\bar{b}_{\alpha}\right) \\
= & \frac{\rho_{\beta}}{1+\rho_{\beta}}\left(b_{\alpha}-\bar{b}_{\alpha}\right)<b_{\alpha}-\bar{b}_{\alpha} .
\end{aligned}
$$


The second equality holds by using because $a<2 a-b_{\alpha}<2 a$ by $b_{\alpha} \in\left[a \rho_{\beta}, a\right), 8 a^{2}>4 a^{2}>\left(2 a-b_{\alpha}\right)$ $(\sqrt{m}+\sqrt{n})(\sqrt{m}-\sqrt{n})=m-n$; the first inequality is true $\quad 2$, and then

$$
\begin{aligned}
& \sqrt{\left(2 a-b_{\alpha}\right)^{2}+8 a^{2}\left(\rho_{\beta}^{2}+2 \rho_{\beta}\right)}>\sqrt{\left(2 a-b_{\alpha}\right)^{2}+\left(2 a-b_{\alpha}\right)^{2}\left(\rho_{\beta}^{2}+2 \rho_{\beta}\right)}, \\
& =\left(2 a-b_{\alpha}\right)\left(1+\rho_{\beta}\right) .
\end{aligned}
$$

Proof of Proposition 5.

(i) If $v_{\beta}>v_{\alpha}$, we have $v_{\beta}-b_{\beta}>v_{\alpha}-b_{\alpha}$, since $b_{\alpha} \geq b_{\beta}$ that is assumed throughout the paper. Thus, the auction is always efficient.

(ii) If $v_{\alpha}>v_{\beta}$. Substituting (14) into $v_{\alpha}-b_{\alpha}<(>) v_{\beta}-b_{\beta}$ and rearranging, we have $v_{\alpha}-v_{\beta}<(>) 2\left(a \rho_{\alpha}-b_{\beta}\right) /$ $\left(2+\rho_{\alpha}\right)$. Substituting (17) into $v_{\alpha}-b_{\alpha}<(>) v_{\beta}-b_{\beta}$ and rearranging, we have $v_{\alpha}-v_{\beta}<(>) p\left(b_{\alpha}\right)$. This completes the proof.

\section{Proof of Proposition 6.}

(i) Since the auction in Model I is inefficient. Equivalently, we have $v_{\alpha}-v_{\beta}<2\left(a \rho_{\alpha}-b_{\beta}\right) /\left(2+\rho_{\alpha}\right)$ by Proposition 5 (ii). When bidder $\beta$ becomes more (less) risk averse, we have $2\left(a \rho_{\alpha}-b_{\beta}\right) /\left(2+\rho_{\alpha}\right)<$ $(>) 2\left(a \rho_{\alpha}-\widehat{b}_{\beta}\right) /\left(2+\rho_{\alpha}\right)$ since $\widehat{b}_{\beta}<(>) b_{\beta}$ by Proposition 3 (i). Thus, the auction may become more inefficient (may be efficient or may be not).

Suppose that the auction in Model I is efficient. Equivalently, we have $v_{\alpha}-v_{\beta}>2\left(a \rho_{\alpha}-b_{\beta}\right) /\left(2+\rho_{\alpha}\right)$ by Proposition 5 (ii). When bidder $\beta$ becomes more (less) risk averse, we have $2\left(a \rho_{\alpha}-b_{\beta}\right) /\left(2+\rho_{\alpha}\right)<$ $(>) 2\left(a \rho_{\alpha}-\widehat{b}_{\beta}\right) /\left(2+\rho_{\alpha}\right)$ since $\widehat{b}_{\beta}<(>) b_{\beta}$ by Proposition 3 (i). Thus, the auction may be efficient or may be not (is still efficient).

(ii) Since the auction in Model II is inefficient. Equivalently, we have $v_{\alpha}-v_{\beta}<p\left(b_{\alpha}\right)$ by Proposition 5 (ii). Using (26), we have

$$
p^{\prime}\left(b_{\alpha}\right)=\frac{1}{2+\rho_{\beta}}\left(1-\frac{\left(2 a-b_{\alpha}\right)}{\sqrt{\left(2 a-b_{\alpha}\right)^{2}+8 a^{2}\left(\rho_{\beta}^{2}+2 \rho_{\beta}\right)}}\right)>0 \text {. }
$$

When bidder $\alpha$ becomes more (less) risk averse, from Proposition 4 (i), the authors have $\bar{b}_{\alpha}<(>) b_{\alpha}$, implying $p\left(\bar{b}_{\alpha}\right)<(>) p\left(b_{\alpha}\right)$. Thus, $v_{\alpha}-v_{\beta}<p\left(b_{\alpha}\right)<p\left(\bar{b}_{\alpha}\right)$ and then the auction in Model II becomes more inefficient if bidder $\alpha$ becomes less risk averse. But when the bidder $\alpha$ becomes more risk averse the auction may be efficient or may be not since the authors cannot conclude the inequality $v_{\alpha}-v_{\beta}>p\left(\bar{b}_{\alpha}\right)$.

Suppose that the auction in Model II is efficient. Equivalently, the authors have $v_{\alpha}-v_{\beta}>p\left(b_{\alpha}\right)$ by Proposition 5 (ii). Since $p^{\prime}\left(b_{\alpha}\right)>0$, when bidder $\alpha$ becomes more (less) risk averse, from Proposition 4 (i), the authors have $\bar{b}_{\alpha}<(>) b_{\alpha}$, implying $p\left(\bar{b}_{\alpha}\right)<(>) p\left(b_{\alpha}\right)$. Thus, $v_{\alpha}-v_{\beta}>p$ $\left(b_{\alpha}\right)>p\left(\bar{b}_{\alpha}\right)$ and then the auction in Model II is still efficient if bidder $\alpha$ becomes more risk averse. But when the bidder $\alpha$ becomes less risk averse, the auction may be efficient or may be not since the authors cannot conclude the inequality $v_{\alpha}-v_{\beta}>p\left(\bar{b}_{\alpha}\right)$

\section{Proof of Proposition 7.}

Consider Model I. Substituting (14) into (28), the authors obtain the expected revenue of the seller $E_{R}\left(b_{\beta}\right)=V$ $\left(b_{\beta}\right)$, where

$$
V(x)=\frac{1}{4 a^{2}}\left(\frac{4}{3} a^{3}+4 \mu a^{2}-2 a^{2}\left(\frac{\rho_{\alpha}(2 a+x)}{2+\rho_{\alpha}}+x\right)+a\left(\frac{\rho_{\alpha}(2 a+x)}{2+\rho_{\alpha}}-x\right)^{2}-\frac{1}{6}\left(\frac{\rho_{\alpha}(2 a+x)}{2+\rho_{\alpha}}-x\right)^{3}\right) .
$$

Similarly, when the bidder $\beta$ becomes more risk averse and has a utility function $\widehat{u}_{\beta}$, the expected revenue of the seller is $\widehat{E}_{R}\left(\widehat{b}_{\beta}\right)=V\left(\widehat{b}_{\beta}\right)$.

$$
E_{R}\left(b_{\beta}\right)-\widehat{E}_{R}\left(\widehat{b}_{\beta}\right)=\frac{\left(b_{\beta}-\widehat{b}_{\beta}\right)}{12 a^{2}\left(2+\rho_{\alpha}\right)^{3}} h\left(\widehat{b}_{\beta}, b_{\beta}\right),
$$

where $h\left(\widehat{b}_{\beta}, b_{\beta}\right)=4 \widehat{b}_{\beta}^{2}+\left(4 b_{\beta}+12 a-6 a \rho_{\alpha}\right) \widehat{b}_{\beta}+4 b_{\beta}^{2}+(12 a-$ $\left.6 a \rho_{\alpha}\right) b_{\beta}-16 a^{2}-56 a^{2} \rho_{\alpha}-20 a^{2} \rho_{\alpha}^{2}-4 a^{2} \rho_{\alpha}^{3}, b_{\beta}, b_{\beta} \in\left(0, a \rho_{\alpha}\right]$ and $\rho_{\alpha} \in(0,1]$.

The authors have $4 b_{\beta}+12 a-6 a \rho_{\alpha}>0,12 a-6 a \rho_{\alpha}>0$ for $b_{\beta} \in\left(0, a \rho_{\alpha}\right], \rho_{\alpha} \in(0,1]$. When $\widehat{b}_{\beta}, b_{\beta}=a \rho_{\alpha}$, the authors $\operatorname{obtain} h\left(a \rho_{\alpha}, a \rho_{\alpha}\right)=-16 a^{2}-32 a^{2} \rho_{\alpha}-20 a^{2} \rho_{\alpha}^{2}-4 a^{2} \rho_{\alpha}^{3}<0$. Then, $h\left(b_{\beta}, b_{\beta}\right)<0$. Since $b_{\beta}>\widehat{b}_{\beta}$. Thus, the authors have $E_{R}<\widehat{E}_{R}$.

Consider Model II. When bidder $\alpha$ becomes more risk averse, the expected revenue of the seller is

$$
\bar{E}_{R}=\frac{1}{4 a^{2}}\left(\frac{4}{3} a^{3}+4 \mu a^{2}-2 a^{2}\left(\bar{b}_{\alpha}+\bar{b}_{\beta}\right)+a\left(\bar{b}_{\alpha}-\bar{b}_{\beta}\right)^{2}-\frac{1}{6}\left(\bar{b}_{\alpha}-\bar{b}_{\beta}\right)^{3}\right) .
$$

By (28), the authors obtain 


$$
E_{R}-\bar{E}_{R}=\frac{1}{4 a^{2}}\left(2 a^{2}\left(\bar{b}_{\alpha}-b_{\alpha}+\bar{b}_{\beta}-b_{\beta}\right)+a\left(\left(b_{\alpha}-b_{\beta}\right)^{2}-\left(\bar{b}_{\alpha}-\bar{b}_{\beta}\right)^{2}\right)+\frac{1}{6}\left(\bar{b}_{\alpha}-\bar{b}_{\beta}\right)^{3}-\frac{1}{6}\left(b_{\alpha}-b_{\beta}\right)^{3}\right)
$$

By simplifying, the authors have

$$
\begin{aligned}
E_{R}-\bar{E}_{R}= & \frac{1}{4 a^{2}}\left(2 a^{2}\left(\bar{b}_{\alpha}-b_{\alpha}+\bar{b}_{\beta}-b_{\beta}\right)+\left(b_{\alpha}-b_{\beta}\right)^{2}\left(a-\frac{1}{6}\left(b_{\alpha}-b_{\beta}\right)\right)+\left(\bar{b}_{\alpha}-\bar{b}_{\beta}\right)^{2}\left(\frac{1}{6}\left(\bar{b}_{\alpha}-\bar{b}_{\beta}\right)-a\right),\right. \\
& <\left(b_{\alpha}-b_{\beta}\right)^{2}\left(a-\frac{1}{6}\left(b_{\alpha}-b_{\beta}\right)\right)+\left(\bar{b}_{\alpha}-\bar{b}_{\beta}\right)^{2}\left(\frac{1}{6}\left(\bar{b}_{\alpha}-\bar{b}_{\beta}\right)-a\right) \\
= & \left(a-\frac{1}{6}\left(b_{\alpha}-b_{\beta}\right)\right)\left(\left(b_{\alpha}-b_{\beta}\right)^{2}-\left(\bar{b}_{\alpha}-\bar{b}_{\beta}\right)^{2}\right) \\
& <\left(a-\frac{1}{6}\left(b_{\alpha}-b_{\beta}\right)\right)\left(b_{\alpha}-b_{\beta}+\bar{b}_{\alpha}-\bar{b}_{\beta}\right)\left(b_{\alpha}-b_{\beta}-\bar{b}_{\alpha}+\bar{b}_{\beta}\right)<0,
\end{aligned}
$$

where the first inequality follows from $\bar{b}_{\alpha}<b_{\alpha}$ and $\bar{b}_{\beta}<b_{\beta}$ (Proposition 4) and the second one holds since $\bar{b}_{\alpha}-\bar{b}_{\beta}<b_{\alpha}-b_{\beta}($ Proposition 4$)$.

\section{Data Availability}

The data used to support the findings of this study are included within the article.

\section{Conflicts of Interest}

The authors declare that they have no conflicts of interest regarding the publication of this paper.

\section{Acknowledgments}

This work was supported by the National Natural Science Foundation of China under Grant no. 71571044.

\section{References}

[1] B. Lebrun, "Existence of an equilibrium in first price auctions," Economic Theory, vol. 7, no. 3, pp. 421-443, 1996.

[2] E. Maskin and J. Riley, "Equilibrium in sealed high bid auctions," The Review of Economic Studies, vol. 67, no. 3, pp. 439-454, 2000a.

[3] E. Maskin and J. Riley, "Asymmetric auctions," The Review of Economic Studies, vol. 67, no. 3, pp. 413-438, 2000.

[4] R. Kirkegaard, "Asymmetric first price auctions," Journal of Economic Theory, vol. 144, no. 4, pp. 1617-1635, 2009.

[5] T. R. Kaplan and S. Zamir, "Asymmetric first-price auctions with uniform distributions: analytic solutions to the general case," Economic Theory, vol. 50, no. 2, pp. 269-302, 2012.

[6] J. G. Riley and W. F. Samuelson, "Optimal auctions," The American Economic Review, vol. 71, no. 3, pp. 381-392, 1981.

[7] S. Matthews, "Comparing auctions for risk averse Buyers: a Buyer's point of view," Econometrica, vol. 55, no. 3, pp. 633-646, 1987.

[8] A. Hu, S. A. Matthews, and L. Zou, "Risk aversion and optimal reserve prices in first- and second-price auctions," Journal of Economic Theory, vol. 145, no. 3, pp. 1188-1202, 2010.
[9] F. Maréchal and P.-H. Morand, "First-price sealed-bid auctions when bidders exhibit different attitudes toward risk," Economics Letters, vol. 113, no. 2, pp. 108-111, 2011.

[10] T. von Ungern-Sternberg, "Swiss auctions," Economica, vol. 58, no. 231, pp. 341-357, 1991.

[11] P. Klemperer, "Auction theory: a guide to the literature," Journal of Economic Surveys, vol. 13, no. 3, pp. 227-286, 1999.

[12] V. Krishna, Auction Theory, Academic Press, 2002. 